ESAIM: COCV 20 (2014) 524-546

DOI: $10.1051 / \mathrm{cocv} / 2013074$
ESAIM: Control, Optimisation and Calculus of Variations

www.esaim-cocv.org

\title{
DUAL-WEIGHTED GOAL-ORIENTED ADAPTIVE FINITE ELEMENTS FOR OPTIMAL CONTROL OF ELLIPTIC VARIATIONAL INEQUALITIES *
}

\author{
M. HintermülleR ${ }^{1}$, R.H.W. Hoppe ${ }^{2,3}$ AND C. LÖBhARD ${ }^{1}$
}

\begin{abstract}
A dual-weighted residual approach for goal-oriented adaptive finite elements for a class of optimal control problems for elliptic variational inequalities is studied. The development is based on the concept of C-stationarity. The overall error representation depends on primal residuals weighted by approximate dual quantities and vice versa as well as various complementarity mismatch errors. Also, a priori bounds for C-stationary points and associated multipliers are derived. Details on the numerical realization of the adaptive concept are provided and a report on numerical tests including the critical cases of biactivity are presented.
\end{abstract}

Mathematics Subject Classification. 49M25, 65K15, 90C33.

Received September 28, 2012. Revised June 24, 2013.

Published online March 27, 2014.

\section{INTRODUCTION}

In this paper we study goal-oriented adaptive finite element methods for a class of optimal control problems with variational inequality constraints. The latter problem class falls into the broader category of mathematical programs with equilibrium constraints, or MPECs for short, which, due to constraint degeneracy, cannot be treated by qualified Karush-Kuhn-Tucker-type theory for the derivation of stationarity systems; see $[28,35]$ in finite dimensions and $[4,30,31,33]$ in function space. The associated difficulties result from so-called biactivities in a regular setting, i.e. a situation where certain multipliers admit a pointwise interpretation, or - in the terminology of optimal control - the fact that the control-to-state mapping is in general only directionally

\footnotetext{
Keywords and phrases. Adaptive finite element method, C-stationarity, goal-oriented error estimation, mathematical programming with equilibrium constraints, optimal control of variational inequalities.

* M.H. acknowledges support by the German Research Fund (DFG) through the Research Center MATHEON Project C28 and C31 and the SPP 1253 "Optimization with Partial Differential Equations", the Austrian Science Fund (FWF) through the START Project Y 305-N18 "Interfaces and Free Boundaries" and the SFB Project F32 04-N18 "Mathematical Optimization and Its Applications in Biomedical Sciences", and support through a J. Tinsely Oden Fellowship at the Institute for Computational Engineering and Sciences (ICES) at UT Austin, Texas, USA. R.H. has been supported by NSF-DMS 1115658, by the DFG-SPP 1253 and DFG-SPP 1506, by the BMBF projects "FROPT" and "MeFreSim", and by the ESF within the Research Networking Programme "OPTPDE". C.L. was supported by the DFG through SPP 1253 "Optimization with Partial Differential Equations".

1 Department of Mathematics, Humboldt-Universität zu Berlin, Unter den Linden 6, 10099 Berlin, Germany. hint@math.hu-berlin.de; loebhard@math.hu-berlin.de

2 Department of Mathematics, University of Houston, Houston, TX 77204-3008, USA. rohop@math.uh.edu

3 Institute of Mathematics, University of Augsburg, Universitätsstraße 14, 86152 Augsburg, Germany.

hoppe@math.uni-augsburg.de
} 
differentiable. The latter fact renders the problem under investigation non-convex and non-differentiable. This structural property is responsible not only for the aforementioned theoretical challenges in describing stationarity, but it also causes problems in the design of reliable solution algorithms; see, e.g., the discussion in $[14,25,35]$ in finite dimensions and [20-22] in function space.

As our underlying problem class is originally posed in function space, the need for discretization for its numerical realization arises naturally. In this context one is interested in obtaining a specified accuracy in the approximate solution or a pre-defined target quantity by the least possible computational effort. Besides the development of efficient solvers, this concerns in particular the design of adaptive finite element methods for the discretization of the continuous problem or stationarity system. Based on a suitable error estimator or indicator, the latter technique aims at refining the discretization locally only in regions with large errors while keeping elements coarse wherever possible. This procedure ideally minimizes the degrees of freedom for computing an approximate solution to the original problem in order to achieve a user-specified accuracy.

Over the years different approaches to adaptive finite element methods (AFEMs) for the numerical treatment of partial differential equations have been studied. Here we only refer to the monographs $[8,11,37,42]$ and the many references therein. By now, a high level of sophistication has been reached. In contrast to this development, AFEM for elliptic variational inequalities, though well investigated, has not yet reached a comparable level; see $[5,6,9,19,24,34,41]$ and the references therein. This is due to the presence of the coincidence or active set with respect to the solution of the variational inequality, which, in general, causes a non-smooth dependence of the solution on the data and, thus, complicates the numerical treatment significantly. Recently, AFEM was successfully carried over to control and/or state constrained optimal control problems (not MPECs!); compare $[10,15-18,29,39,43]$. In particular, in $[10,15-18,43]$ the dual-weighted residual (DWR) approach to goal-oriented adaptivity, which was pioneered by [7] (see also the monograph [8]), was extended to the discretization of control and/or state constrained optimal control problems with partial differential equation constraints.

In the present paper, we develop a goal-oriented mesh refinement technique in the spirit of the DWR approach for the adaptive discretization of MPECs in function space. Based on the notion of a modified Lagrangian function associated with the MPEC or more precisely mathematical program with complementarity constraints (MPCC, for short), the so-called MPCC-Lagrangian, an error estimator (indicator) is derived which involves primal residuals weighted by dual variables and vice versa as well as error terms covering the mismatch in complementarity. The latter error indicators are relevant in the location of the coincidence sets which arise due to the variational inequality constraint. Finally, we point out that the literature on goal-oriented mesh adaptivity for MPCCs or MPECs in function space, and in particular for the problem class under investigation, appears essentially void.

The rest of this text is organized as follows. In Section 2 we outline the problem class under consideration in the original continuous form and also in its discrete version, and provide basic assertions as optimality systems and a priori estimates. Section 3 gives an error representation, which turns out to be a combination of weighted residuals and products reflecting the mismatch in complementarity. Although this representation is not an estimator, i.e., it depends on the (unknown) solutions of the continuous problem, it guides a way to an a posteriori error indicator. In Section 4, we derive fully a posteriori indicators for the error contribution on every element of the discretization. The last Section 5 provides the basic algorithms used in our numerical solver and reports on numerical results for some test problems.

Notation. Throughout this text, let $\Omega \subset \mathbb{R}^{n}$ be a bounded domain with $\partial \Omega$ denoting its boundary. We write $(z, w)_{0, \Omega}$ for the canonical scalar product in the Lebesgue space $L^{2}(\Omega)$, and $\|\cdot\|_{0, \Omega}$ for the induced norm. For $n \in\{1,2,3\}, H^{1}(\Omega)$ is the Sobolev space of $L^{2}(\Omega)$-functions with gradients in $L^{2}(\Omega)^{n}$ and $H_{0}^{1}(\Omega):=\{y \in$ $\left.H^{1}(\Omega)|y|_{\partial \Omega}=0\right\}$ endowed with the norm $\|z\|_{1, \Omega}:=\|\nabla z\|_{0, \Omega}$.

For a function $z \in H^{1}(\Omega)$ and a set $\omega \subset \bar{\Omega}$, we say that $\left.z\right|_{\omega} \geq 0$ in the sense of $H^{1}(\Omega)$, if there exists a sequence $\left(z_{n}\right)_{n \in \mathbb{N}} \subset W^{1, \infty}(\Omega)$ such that for all $x \in \omega, z_{n}(x) \geq 0$ and $z_{n} \rightarrow z$ in $H^{1}(\Omega)$. Whenever $\omega=\Omega$, then we simply write $y \geq 0$. With this definition, the relations " $\leq$ " and "=" on subsets of $\bar{\Omega}$ can be defined 
canonically on $H^{1}(\Omega)$ (cf. [26], p. 35). For the relation of non-negativity in the sense of $H^{1}(\Omega)$ and the notion of non-negativity in the almost everywhere sense we refer to ([26], Prop. II.5.2). We abbreviate, for example, $\{z \geq 0\}:=\{x \in \Omega \mid z(x) \geq 0\}$ and, for functions $z \in L^{2}(\Omega)$, the above set is defined only up to a set of measure zero.

The dual space of $H_{0}^{1}(\Omega)$ is denoted by $H^{-1}(\Omega)$, and for $\lambda \in H^{-1}(\Omega)$ and $z \in H_{0}^{1}(\Omega)$ the dual pairing is written as $\langle\lambda, z\rangle_{-1,1, \Omega}:=\lambda(z)$. For functionals $z \in H^{-1}(\Omega)$ and a subset $\omega \subset \Omega$, we write $\left.z\right|_{\omega}=0$, if $\forall \phi \in H_{0}^{1}(\Omega)$ with $\left.\phi\right|_{\Omega \backslash \omega}=0$, it holds that $\langle z, \phi\rangle_{-1,1, \Omega}=0$, and we write $\left.z\right|_{\omega} \geq 0$, if $\forall \phi \in H_{0}^{1}(\Omega)$ with $\left.\phi\right|_{\Omega \backslash \omega}=0$ and $\left.\phi\right|_{\Omega \backslash \omega} \geq 0$, it holds that $\langle z, \phi\rangle_{-1,1, \Omega} \geq 0$.

In the rest of the paper, we simply use the symbol " $\geq$ ". For $L^{2}(\Omega)$-functions it refers to " $\geq$ " in the almost everywhere (a.e.) sense, for $H^{1}(\Omega)$-functions and $H^{-1}(\Omega)$-objects it is to be understood in the respective sense as described above. In particular, for $\lambda \in H^{-1}(\Omega), \lambda \geq 0$ refers to $\langle\lambda, z\rangle_{1,-1, \Omega} \geq 0$ for all $z \in H_{0}^{1}(\Omega)$.

\section{Statement of the Problem And Stationarity}

\subsection{Problem definition}

We consider the optimal control problem with a variational inequality constraint given by

$$
\begin{aligned}
& \text { Minimize } \quad J(y, u):=\frac{1}{2}\left\|y-y_{d}\right\|_{0, \Omega}^{2}+\frac{\nu}{2}\|u\|_{0, \Omega}^{2} \\
& \text { over } \quad(y, u) \in H_{0}^{1}(\Omega) \times L^{2}(\Omega), \\
& \text { subject to } \\
& \begin{aligned}
y & \geq 0, \\
\forall z & \in H_{0}^{1}(\Omega), \quad z \geq 0, \quad a(y, z-y) \geq(u+f, z-y)_{0, \Omega},
\end{aligned}
\end{aligned}
$$

i.e. we minimize an objective functional $J(y, u)$ depending on a state variable $y \in H_{0}^{1}(\Omega)$ with homogeneous Dirichlet boundary conditions and a control variable $u \in L^{2}(\Omega)$, both defined on a bounded domain $\Omega \subset \mathbb{R}^{n}$, $n \in\{1,2,3\}$. The given function $y_{d} \in L^{2}(\Omega)$ is the desired state and $\nu>0$ is the cost of the control action.

The constraint involves on the one hand an obstacle $\psi \equiv 0$ which bounds the state $y$ from below, i.e., $y \geq 0$, and on the other hand a bounded, $H_{0}^{1}(\Omega)$-elliptic bilinear form $a: H_{0}^{1}(\Omega) \times H_{0}^{1}(\Omega) \rightarrow \mathbb{R}$ which describes the influence of forces, such as the fixed volume force $f \in L^{2}(\Omega)$ and the action of the control $u$, applied to the state $y$. We mention that (2.1) models the control of the deflection ( $y$ from an equilibrium) of a membrane covering the domain $\Omega$ (and fixed on the boundary $\partial \Omega$ ), under a given loading $f$ and a fixed rigid obstacle $\psi \equiv 0$ yielding $y \geq \psi \equiv 0$, towards a desired displacement profile $y_{d}$, where $\nu>0$ reflects the $L^{2}(\Omega)$-averaged cost of the control action. This model can be seen as a simplified version of a control problem for the contact of an elastic body with a rigid obstacle.

The existence of a solution to problem (2.1) can be shown by arguments based on infimizing sequences; see, e.g., $[4,32]$.

Remark 2.1. We note that considering $\psi \equiv 0$ does not imply restrictions with respect to the choice of the obstacle aside from $H_{0}^{1}(\Omega)$-regularity.

In the formulation of the first order optimality system and in the numerical solver for the optimal control problem, a reformulation of the variational inequality (2.1d) by means of an additional slack variable $\xi$ is considered. For this purpose, let $y \in H_{0}^{1}(\Omega), y \geq 0$ and $u \in L^{2}(\Omega)$, and introduce the operator $\mathcal{A}: H_{0}^{1}(\Omega) \rightarrow$ $H^{-1}(\Omega)$ which satisfies $a(y, z)=\langle\mathcal{A} y, z\rangle_{-1,1, \Omega}$ for all $y, z \in H_{0}^{1}(\Omega)$ as well as a slack variable $\xi \in H^{-1}(\Omega)$ as follows,

$$
\xi:=\mathcal{A} y-u-f \in H^{-1}(\Omega) .
$$

Then, $(2.1 \mathrm{c})-(2.1 \mathrm{~d})$ is equivalent to

$$
y \geq 0, \quad \xi \geq 0 \quad \text { and } \quad\langle\xi, y\rangle_{-1,1, \Omega}=0 .
$$


Thus, for given data $f, y_{d} \in L^{2}(\Omega)$ on a domain $\Omega$ and $\nu>0$, problem (2.1) can be rewritten as an optimal control problem with a partial differential equation and a complementarity constraint as follows: find a solution $(y, u, \xi) \in H_{0}^{1}(\Omega) \times L^{2}(\Omega) \times H^{-1}(\Omega)$ to the problem

$$
\begin{aligned}
& \text { Minimize } \quad J(y, u)=\frac{1}{2}\left\|y-y_{d}\right\|_{0, \Omega}^{2}+\frac{\nu}{2}\|u\|_{0, \Omega}^{2} \\
& \text { subject to } \quad a(y, z)=(u+f, z)_{0, \Omega}+\langle\xi, z\rangle_{-1,1, \Omega}, \quad \forall z \in H_{0}^{1}(\Omega) \text {, } \\
& y \geq 0 \text { in } H_{0}^{1}(\Omega), \xi \geq 0 \text { in } H^{-1}(\Omega) \text { and }\langle\xi, y\rangle_{-1,1, \Omega}=0 .
\end{aligned}
$$

Due to the complementarity structure of $(2.2 \mathrm{c})$ we call $(2.2)$ a mathematical program with complementarity constraints, or an MPCC, for short.

Remark 2.2 (regularity of the data). In the situation of ([38], Prop. 5:2.2), where $\mathcal{A}: W \rightarrow V^{\prime}$ represents a strictly T-monotone operator, we set $W=H_{0}^{1}(\Omega)$, which is a vector lattice and embeds continuously into $H=L^{2}(\Omega)$, and $V^{\prime}=H^{-1}(\Omega)$. Then we have $\left\|\mathcal{A} y^{\star}\right\|_{0, \Omega} \leq C_{\mathrm{R}}(\Omega)\left(\|f\|_{0, \Omega}+\left\|u^{\star}\right\|_{0, \Omega}\right)$ for the solution $y^{\star}$ of the variational inequality in (2.1d) with control variable $u^{\star} \in L^{2}(\Omega)$. Thus, if for instance $\mathcal{A}=-\Delta$ on a convex domain $\Omega$ (or a domain with boundary $\partial \Omega$ of class $C^{1,1}$ ), then $y^{\star} \in H_{0}^{1}(\Omega) \cap H^{2}(\Omega)$ (cf. [38], Cor. 5:2.3). Moreover, in the reformulation resulting in the linear complementarity problem $(2.2 \mathrm{~b})-(2.2 \mathrm{c})$ it holds that $\xi^{\star} \in L^{2}(\Omega)$.

\subsection{Stationarity conditions}

In the error analysis in Section 3, we use stationarity conditions which are weaker than those stated in [32], but yet much stronger than those obtained in [4]. While one can guarantee strong stationarity for a solution of (2.2) according to [32], here we only use the weaker notion of $C$ (larke)-stationarity of the solution as only C-stationary points can usually be obtained with guarantee by current state-of-the-art solution algorithms, see [21, 23, 27]. For the aforementioned categorization of stationarity in function space, we refer to [21]. Obviously, the available stationarity condition is also essential for the development of a posteriori error estimators. For a more general definition of finite dimensional variants of stationarity concepts we refer to [40]. Function space versions of C-stationarity conditions in the regular case of Remark 2.2, which are more restrictive than the versions stated here, can be found in [21].

Definition 2.3 (Clarke or C-stationarity). A point $(y, u, \xi) \in H_{0}^{1}(\Omega) \times L^{2}(\Omega) \times H^{-1}(\Omega)$ is called C-stationary for problem (2.2), if there exist $p \in H_{0}^{1}(\Omega), \lambda \in H^{-1}(\Omega)$ and $\mu \in H_{0}^{1}(\Omega)$ such that the following conditions hold,

$$
\begin{aligned}
\mathcal{A} y-u-\xi & =f & & \text { in } H^{-1}(\Omega), \\
\xi \geq 0, y \geq 0,\langle\xi, y\rangle_{-1,1, \Omega} & =0, & & \\
y-\lambda+\mathcal{A}^{*} p & =y_{d} & & \text { in } H^{-1}(\Omega), \\
\nu u-p & =0 & & \text { in } H_{0}^{1}(\Omega), \\
-\mu-p & =0 & & \text { in } H_{0}^{1}(\Omega), \\
\langle\lambda, y\rangle_{-1,1, \Omega} & =0, & & \\
\langle\xi, \mu\rangle_{-1,1, \Omega} & =0, & & \\
\langle\lambda, \mu\rangle_{-1,1, \Omega} & \geq 0 . & &
\end{aligned}
$$

Definition 2.4 (strong stationarity). A point $(y, u, \xi) \in H_{0}^{1}(\Omega) \times L^{2}(\Omega) \times H^{-1}(\Omega)$ is called strongly stationary for problem (2.2), if it is C-stationary and if the multipliers $\lambda \in H^{-1}(\Omega)$ and $\mu \in H_{0}^{1}(\Omega)$ in Definition 2.3 satisfy the sign conditions

$$
\forall \psi \in H_{0}^{1}(\Omega),\left.\psi\right|_{\{y=0\}} \geq 0 \text { and }\langle\xi, \psi\rangle_{-1,1, \Omega}=0: \quad \begin{aligned}
\langle\lambda, \psi\rangle_{-1,1, \Omega} & \geq 0, \\
\left.\mu\right|_{\{y=0\}} & \geq 0 .
\end{aligned}
$$


We introduce $X$ as the function space $X:=H_{0}^{1}(\Omega) \times L^{2}(\Omega) \times H^{-1}(\Omega) \times H_{0}^{1}(\Omega)$ with elements $x=(y, u, \xi, p)$, and define the MPCC-Lagrangian $\mathcal{L}: X \times H^{-1}(\Omega) \times H_{0}^{1}(\Omega) \rightarrow \mathbb{R}$ according to

$$
\begin{aligned}
\mathcal{L}(y, u, \xi, p, \lambda, \mu):= & J(y, u)+a(y, p)-(u+f, p)_{0, \Omega}-\langle\xi, p\rangle_{-1,1, \Omega} \\
& -\langle\lambda, y\rangle_{-1,1, \Omega}-\langle\xi, \mu\rangle_{-1,1, \Omega} .
\end{aligned}
$$

Remark 2.5. We note that if $\left(y^{\star}, u^{\star}, \xi^{\star}\right)$ is C-stationary and $p^{\star}, \lambda^{\star}, \mu^{\star}$ are the associated dual variables from (2.3), then

$$
\mathcal{L}\left(x^{\star}, \lambda^{\star}, \mu^{\star}\right)=J\left(y^{\star}, u^{\star}\right),
$$

and for all $\delta x \in X$ it holds that

$$
\nabla_{x} \mathcal{L}\left(x^{\star}, \lambda^{\star}, \mu^{\star}\right)(\delta x)=0 .
$$

Remark 2.6. The functional $\mathcal{L}\left(\cdot, \lambda^{\star}, \mu^{\star}\right): X \rightarrow \mathbb{R}$ is infinitely Gâteaux differentiable at $x \in X$. The second Gâteaux derivative $\nabla_{x x} \mathcal{L}\left(x, \lambda^{\star}, \mu^{\star}\right)$ is independent of the argument $\left(x, \lambda^{\star}, \mu^{\star}\right)$ which for notational convenience will be omitted in the sequel.

\subsection{A priori estimates for solutions of the optimality system}

This section is devoted to an a priori analysis of the dependence of a C-stationary point $\left(y^{\star}, u^{\star}, \xi^{\star}\right)$ with dual variables $p^{\star}, \lambda^{\star}$ and $\mu^{\star}$ on the problem input data $\Omega, f, y_{d}$, and $\nu$. The following result states a priori bounds which are, for instance, relevant in the study of stability of solutions with respect to data perturbations.

For its formulation we invoke the following assumption concerning the data involved in (2.1). The coercive bilinear form $a: H_{0}^{1}(\Omega) \times H_{0}^{1}(\Omega) \rightarrow \mathbb{R}$ admits the representation $\mathcal{A} \in \mathcal{L}\left(H_{0}^{1}(\Omega), H^{-1}(\Omega)\right)$ with coercivity constant $\Sigma_{\mathcal{A}}>0$ satisfying

$$
a(y, z)=\langle\mathcal{A} y, z\rangle_{-1,1, \Omega} \quad \text { and } \quad a(y, y) \geq \Sigma_{\mathcal{A}}\|y\|_{1, \Omega}^{2}
$$

for all $y, z \in H_{0}^{1}(\Omega)$. Let $C_{\mathcal{A}}(\Omega)=\|\mathcal{A}\|_{\mathcal{L}\left(H_{0}^{1}(\Omega), H^{-1}(\Omega)\right)}$ abbreviate the norm of the operator $\mathcal{A}$, and let $\mathcal{A}^{*}$ denote the adjoint of $\mathcal{A}$. Moreover, we denote the Friedrichs constant for the domain $\Omega$ by $C_{\mathrm{F}}(\Omega)$ and the constant arising from the embedding $L^{2}(\Omega) \hookrightarrow H^{-1}(\Omega)$ by $C_{-1}(\Omega)$, i.e., for all $y \in H_{0}^{1}(\Omega)$ and $u \in L^{2}(\Omega)$, it holds that

$$
\|y\|_{0, \Omega} \leq C_{\mathrm{F}}(\Omega)\|y\|_{1, \Omega} \quad \text { and } \quad\|u\|_{-1, \Omega} \leq C_{-1}(\Omega)\|u\|_{0, \Omega} .
$$

Proposition 2.7. A solution $\left(x^{\star}, \lambda^{\star}, \mu^{\star}\right)$ of the stationarity system (2.3) satisfies the following a priori bounds,

$$
\begin{aligned}
\left\|y^{\star}\right\|_{0, \Omega} & \leq\left\|y_{d}\right\|_{0, \Omega}+\sqrt{\nu}\|f\|_{0, \Omega}, \\
\left\|y^{\star}\right\|_{1, \Omega} & \leq \frac{C_{F}(\Omega)}{\Sigma_{\mathcal{A}}}\left(\frac{1}{\sqrt{\nu}}\left\|y_{d}\right\|_{0, \Omega}+2\|f\|_{0, \Omega}\right), \\
\left\|u^{\star}\right\|_{0, \Omega} & \leq \frac{1}{\sqrt{\nu}}\left\|y_{d}\right\|_{0, \Omega}+\|f\|_{0, \Omega}, \\
\left\|\xi^{\star}\right\|_{-1, \Omega} & \leq\left(\frac{C_{F}(\Omega)}{\Sigma_{\mathcal{A}}} C_{\mathcal{A}}(\Omega)+C_{-1}(\Omega)\right)\left(\frac{1}{\sqrt{\nu}}\left\|y_{d}\right\|_{0, \Omega}+2\|f\|_{0, \Omega}\right), \\
\left\|u^{\star}\right\|_{1, \Omega} & \leq \frac{C_{F}(\Omega)}{\Sigma_{\mathcal{A}} \nu}\left(2\left\|y_{d}\right\|_{0, \Omega}+\sqrt{\nu}\|f\|_{0, \Omega}\right), \\
\left\|\lambda^{\star}\right\|_{-1, \Omega} & \leq\left(\frac{C_{F}(\Omega)}{\Sigma_{\mathcal{A}}} C_{\mathcal{A}}(\Omega)+C_{-1}(\Omega)\right)\left(2\left\|y_{d}\right\|_{0, \Omega}+\sqrt{\nu}\|f\|_{0, \Omega}\right) .
\end{aligned}
$$

Under the additional assumptions stated in Remark 2.2 we obtain the estimate

$$
\left\|\mathcal{A} y^{\star}\right\|_{0, \Omega} \leq C_{R}(\Omega)\left(\frac{1}{\sqrt{\nu}}\left\|y_{d}\right\|_{0, \Omega}+2\|f\|_{0, \Omega}\right) .
$$


Proof. We start by multiplying equations (2.3a) and (2.3c) by $p^{\star}$ and $y^{\star}$, respectively. Then, using the constraints $(2.3 \mathrm{~d})-(2.3 \mathrm{~g})$, we obtain

$$
\begin{aligned}
& a\left(y^{\star}, p^{\star}\right)=\left(u^{\star}, p^{\star}\right)_{0, \Omega}+\left(f, p^{\star}\right)_{0, \Omega}=\nu\left\|u^{\star}\right\|_{0, \Omega}^{2}+\nu\left(f, u^{\star}\right)_{0, \Omega}, \\
& a\left(y^{\star}, p^{\star}\right)=\left(y_{d}, y^{\star}\right)_{0, \Omega}-\left\|y^{\star}\right\|_{0, \Omega}^{2} .
\end{aligned}
$$

Subtracting (2.13) from (2.14), rearranging terms and estimating the inner products one derives

$$
\begin{aligned}
\left\|y^{\star}\right\|_{0, \Omega}^{2}+\nu\left\|u^{\star}\right\|_{0, \Omega}^{2} & =\left(y_{d}, y^{\star}\right)_{0, \Omega}-\nu\left(f, u^{\star}\right)_{0, \Omega} \\
& \leq \frac{1}{2}\left\|y^{\star}\right\|_{0, \Omega}^{2}+\frac{1}{2}\left\|y_{d}\right\|_{0, \Omega}^{2}+\frac{\nu}{2}\left\|u^{\star}\right\|_{0, \Omega}^{2}+\frac{\nu}{2}\|f\|_{0, \Omega}^{2},
\end{aligned}
$$

and thus

$$
\left\|y^{\star}\right\|_{0, \Omega}^{2}+\nu\left\|u^{\star}\right\|_{0, \Omega}^{2} \leq\left\|y_{d}\right\|_{0, \Omega}^{2}+\nu\|f\|_{0, \Omega}^{2} .
$$

This yields the $L^{2}$-bounds for $y^{\star}$ and $u^{\star}$. Next, we multiply equation (2.3a) by $y^{\star}$, recall that $\left\langle\xi^{\star}, y^{\star}\right\rangle_{-1,1, \Omega}=0$ and deduce

$$
\left\|y^{\star}\right\|_{1, \Omega}^{2} \leq \frac{1}{\Sigma_{\mathcal{A}}}\left(\left(u^{\star}, y^{\star}\right)_{0, \Omega}+\left(f, y^{\star}\right)_{0, \Omega}\right) \leq \frac{C_{\mathrm{F}}(\Omega)}{\Sigma_{\mathcal{A}}}\left(\left\|u^{\star}\right\|_{0, \Omega}+\|f\|_{0, \Omega}\right)\left\|y^{\star}\right\|_{1, \Omega} .
$$

This, together with (2.15), implies the bound on $\left\|y^{\star}\right\|_{1, \Omega}$, i.e.,

$$
\left\|y^{\star}\right\|_{1, \Omega} \leq \frac{C_{\mathrm{F}}(\Omega)}{\Sigma_{\mathcal{A}}}\left(\left\|u^{\star}\right\|_{0, \Omega}+\|f\|_{0, \Omega}\right) \leq \frac{C_{\mathrm{F}}(\Omega)}{\Sigma_{\mathcal{A}}}\left(\frac{1}{\sqrt{\nu}}\left\|y_{d}\right\|_{0, \Omega}+2\|f\|_{0, \Omega}\right) .
$$

The bound on $\left\|\xi^{\star}\right\|_{-1, \Omega}$ involves the constant $C_{-1}(\Omega)$ arising from the embedding $L^{2}(\Omega) \hookrightarrow H^{-1}(\Omega)$ and the norm $C_{\mathcal{A}}(\Omega)=\|\mathcal{A}\|_{\mathcal{L}\left(H_{0}^{1}(\Omega), H^{-1}(\Omega)\right)}$. In fact, from (2.3a), we obtain

$$
\begin{aligned}
\left\|\xi^{\star}\right\|_{-1, \Omega} & =\left\|\mathcal{A} y^{\star}-u^{\star}-f\right\|_{-1, \Omega} \\
& \leq C_{\mathcal{A}}(\Omega)\left\|y^{\star}\right\|_{1, \Omega}+C_{-1}(\Omega)\left(\left\|u^{\star}\right\|_{0, \Omega}+\|f\|_{0, \Omega}\right) \\
& \leq\left(\frac{C_{\mathrm{F}}(\Omega)}{\Sigma_{\mathcal{A}}} C_{\mathcal{A}}(\Omega)+C_{-1}(\Omega)\right)\left(\frac{1}{\sqrt{\nu}}\left\|y_{d}\right\|_{0, \Omega}+2\|f\|_{0, \Omega}\right) .
\end{aligned}
$$

The adjoint equation $(2.3 \mathrm{c})$ tested with $p^{\star}$ and the sign condition $(2.3 \mathrm{~h})$ yielding

$$
\left\langle\lambda^{\star}, p^{\star}\right\rangle_{-1,1, \Omega}=-\left\langle\lambda^{\star}, \mu^{\star}\right\rangle_{-1,1, \Omega} \leq 0
$$

imply

$$
\left\|p^{\star}\right\|_{1, \Omega}^{2} \leq \frac{1}{\Sigma_{\mathcal{A}}}\left(\left(y_{d}, p^{\star}\right)_{0, \Omega}-\left(y^{\star}, p^{\star}\right)_{0, \Omega}\right),
$$

and in the same way as above,

$$
\nu\left\|u^{\star}\right\|_{1, \Omega}=\left\|p^{\star}\right\|_{1, \Omega} \leq \frac{C_{\mathrm{F}}(\Omega)}{\Sigma_{\mathcal{A}}}\left(2\left\|y_{d}\right\|_{0, \Omega}+\sqrt{\nu}\|f\|_{0, \Omega}\right) .
$$

The bound on $\left\|\lambda^{\star}\right\|_{-1, \Omega}$ is derived analogously to the respective bound on $\xi^{\star}$, i.e., one uses the adjoint equation to obtain

$$
\begin{aligned}
\left\|\lambda^{\star}\right\|_{-1, \Omega} & =\left\|\mathcal{A}^{*} p^{\star}+y^{\star}-y_{d}\right\|_{-1, \Omega} \\
& \leq C_{\mathcal{A}}(\Omega)\left\|p^{\star}\right\|_{1, \Omega}+\left\|y^{\star}\right\|_{-1, \Omega}+\left\|y_{d}\right\|_{-1, \Omega} \\
& \leq C_{\mathcal{A}}(\Omega)\left\|p^{\star}\right\|_{1, \Omega}+C_{-1}(\Omega)\left(\left\|y^{\star}\right\|_{0, \Omega}+\left\|y_{d}\right\|_{0, \Omega}\right) \\
& \leq\left(C_{\mathcal{A}}(\Omega) \frac{C_{\mathrm{F}}(\Omega)}{\Sigma_{\mathcal{A}}}+C_{-1}(\Omega)\right)\left(\sqrt{\nu}\|f\|_{0, \Omega}+2\left\|y_{d}\right\|_{0, \Omega}\right) .
\end{aligned}
$$

This proves the first part of the proposition. 
In the setting of Remark 2.2, we obtain

$$
\left\|\mathcal{A} y^{\star}\right\|_{0, \Omega} \leq C_{\mathrm{R}}(\Omega)\left(\|f\|_{0, \Omega}+\left\|u^{\star}\right\|_{0, \Omega}\right) \leq C_{\mathrm{R}}(\Omega)\left(2\|f\|_{0, \Omega}+\frac{1}{\sqrt{\nu}}\left\|y_{d}\right\|_{0, \Omega}\right) .
$$

This concludes the proof.

\subsection{Discretization of the optimal control problem}

We consider the discretization of the optimal control problem (2.1) by finite dimensional spaces $Y_{h} \subset H_{0}^{1}(\Omega)$ and $U_{h} \subset L^{2}(\Omega)$ for the state $y$ and the control $u$, respectively. Let $\mathcal{N}_{Y_{h}}$ and $\mathcal{N}_{U_{h}}$ be sets with cardinality $\left|\mathcal{N}_{Y_{h}}\right|=\operatorname{dim}\left(Y_{h}\right)$ and $\left|\mathcal{N}_{U_{h}}\right|=\operatorname{dim}\left(U_{h}\right)$. Further, we introduce $\left\{\varphi_{z} \mid z \in \mathcal{N}_{Y_{h}}\right\}$ and $\left\{\psi_{z} \mid z \in \mathcal{N}_{U_{h}}\right\}$ as bases of $Y_{h}$ and $U_{h}$, respectively. Then, a function $y_{h}=\sum_{z \in \mathcal{N}_{Y_{h}}} \mathbf{y}_{\mathbf{h}, z} \varphi_{z} \in Y_{h}$ with $\mathbf{y}_{\mathbf{h}, z} \in \mathbb{R}$ can be identified by its component vector

$$
\mathbf{y}_{\mathbf{h}}=\left(\mathbf{y}_{\mathbf{h}, z}\right)_{z \in \mathcal{N}_{Y_{h}}} \in \mathbb{R}^{\left|\mathcal{N}_{Y_{h}}\right|} \text {. }
$$

Similarly, we identify the function $u_{h}=\sum_{z \in \mathcal{N}_{U_{h}}} \mathbf{u}_{\mathbf{h}, z} \psi_{z} \in U_{h}$ with

$$
\mathbf{u}_{\mathbf{h}}=\left(\mathbf{u}_{\mathbf{h}, z}\right)_{z \in \mathcal{N}_{U_{h}}} .
$$

For the ease of presentation, we assume that the basis of $Y_{h}$ yields $y_{h} \geq 0$ in $\Omega$ if and only if $\mathbf{y}_{\mathbf{h}} \geq 0$. A particular example of a discretization satisfying the above conditions is given in Section 4.1.

Next we define the stiffness matrix $\mathcal{A}_{h} \in \mathbb{R}^{\left|\mathcal{N}_{Y_{h}}\right| \times\left|\mathcal{N}_{Y_{h}}\right|}$ and the mass matrix $\mathcal{M}_{h} \in \mathbb{R}^{\left|\mathcal{N}_{Y_{h}}\right| \times\left|\mathcal{N}_{U_{h}}\right|}$ representing the bilinear form $a$ on $Y_{h}$ and the $L^{2}$-scalar-product of functions in $Y_{h}$ with functions in $U_{h}$, respectively. Hence, the elements of these matrices are given by

$$
\left(\mathcal{A}_{h}\right)_{z \bar{z}}:=a\left(\varphi_{z}, \varphi_{\bar{z}}\right) \quad \text { and } \quad\left(\mathcal{M}_{h}\right)_{z v}:=\left(\varphi_{z}, \psi_{v}\right)_{0, \Omega}
$$

for $z, \bar{z} \in \mathcal{N}_{Y_{h}}$ and $v \in \mathcal{N}_{U_{h}}$. Then, for all $y_{h}, w_{h} \in Y_{h}$ with vector representation $\mathbf{y}_{\mathbf{h}}, \mathbf{w}_{\mathbf{h}}$ and all $u_{h} \in U_{h}$ with vector representation $\mathbf{u}_{\mathbf{h}}$ it holds that

$$
\mathbf{y}_{\mathbf{h}}{ }^{T} \mathcal{A}_{h} \mathbf{w}_{\mathbf{h}}=a\left(y_{h}, w_{h}\right) \quad \text { and } \quad \mathbf{y}_{\mathbf{h}}{ }^{T} \mathcal{M}_{h} \mathbf{u}_{\mathbf{h}}=\left(y_{h}, u_{h}\right)_{0, \Omega} .
$$

Analogously, we define mass matrices $\mathcal{M}_{h}^{Y}$ and $\mathcal{M}_{h}^{U}$ representing the $L^{2}(\Omega)$-inner products in $Y_{h}$ and $U_{h}$, respectively.

The data vectors $\mathbf{f}_{\mathbf{h}}=\left(\mathbf{f}_{\mathbf{h}, z}\right)_{z \in \mathcal{N}_{Y_{h}}}$ and $\mathbf{y}_{\mathbf{d h}}=\left(\mathbf{y}_{\mathbf{d h}, z}\right)_{z \in \mathcal{N}_{Y_{h}}}$ are defined by

$$
\mathbf{f}_{\mathbf{h}, z}=\int_{\Omega} f \varphi_{z} \mathrm{~d} x, \quad \mathbf{y}_{\mathbf{d h}, z}=\int_{\Omega} y_{d} \varphi_{z} \mathrm{~d} x .
$$

In the same way as in Section 2, we introduce a slack variable $\boldsymbol{\xi}_{\mathbf{h}} \in \mathbb{R}^{\left|\mathcal{N}_{Y_{h}}\right|}$ as the vector

$$
\boldsymbol{\xi}_{\mathbf{h}}:=\mathcal{A}_{h} \mathbf{y}_{\mathbf{h}}-\mathcal{M}_{h} \mathbf{u}_{\mathbf{h}}-\mathbf{f}_{\mathbf{h}} .
$$

The error representation in Section 3 requires an interpretation of the vector $\boldsymbol{\xi}_{\mathbf{h}}$ as an element $\xi_{h}$ in $H^{-1}(\Omega)$ such that for all $y_{h} \in Y_{h} \subset H_{0}^{1}(\Omega)$ we have

$$
\mathbf{y}_{\mathbf{h}}^{T} \boldsymbol{\xi}_{\mathbf{h}}=\left\langle\xi_{h}, y_{h}\right\rangle_{-1,1, \Omega}
$$

Thus, for any $\boldsymbol{\xi}_{\mathbf{h}} \in \mathbb{R}^{\left|\mathcal{N}_{Y_{h}}\right|}$ let $\hat{\boldsymbol{\xi}}_{\mathbf{h}}:=\left(\mathcal{M}_{h}^{Y}\right)^{-1} \boldsymbol{\xi}_{\mathbf{h}}$ be the coefficients of the function $\xi_{h}$ in the basis $\left\{\varphi_{z} \mid z \in \mathcal{N}_{Y_{h}}\right\}$, i.e.

$$
\xi_{h}:=\sum_{z \in \mathcal{N}_{Y_{h}}}\left(\left(\mathcal{M}_{h}^{Y}\right)^{-1} \boldsymbol{\xi}_{\mathbf{h}}\right)_{z} \varphi_{z} \in L^{2}(\Omega) \hookrightarrow H^{-1}(\Omega) .
$$


Then it holds that

$$
\mathbf{y}_{\mathbf{h}}^{T} \boldsymbol{\xi}_{\mathbf{h}}=\mathbf{y}_{\mathbf{h}}^{T} \mathcal{M}_{h}^{Y}\left(\mathcal{M}_{h}^{Y}\right)^{-1} \boldsymbol{\xi}_{\mathbf{h}}=\mathbf{y}_{\mathbf{h}}^{T} \mathcal{M}_{h}^{Y} \hat{\boldsymbol{\xi}}_{\mathbf{h}}=\left(\xi_{h}, y_{h}\right)_{0, \Omega}=\left\langle\xi_{h}, y_{h}\right\rangle_{-1,1, \Omega}
$$

In the following, we identify the vector $\boldsymbol{\xi}_{\mathbf{h}}$ (rather than $\hat{\boldsymbol{\xi}}_{\mathbf{h}}$ ) with the function $\xi_{h}$ and denote the associated discrete space by $\Xi_{h}\left(=Y_{h}\right)$.

Consider an operator $\Pi$ on $L^{2}(\Omega)$ which satisfies

$$
\left(\Pi v, v_{h}\right)_{0, \Omega}=\left(v, v_{h}\right)_{0, \Omega} \quad \text { for all } v \in L^{2}(\Omega) \text { and } v_{h} \in Y_{h} .
$$

The last step to the formulation of the discrete version of the optimal control problem considered in this text is the definition of the discrete objective functional $J_{\Pi}: H_{0}^{1}(\Omega) \times L^{2}(\Omega) \rightarrow \mathbb{R}$. Later, we will also use the discrete MPCC-Lagrangian $\mathcal{L}_{\Pi}: X \times H^{-1}(\Omega) \times H_{0}^{1}(\Omega) \rightarrow \mathbb{R}$. Thus, we define

$$
\begin{aligned}
J_{\Pi}(y, u):= & \frac{1}{2}\left\|y-\Pi y_{d}\right\|_{0, \Omega}^{2}+\frac{\nu}{2}\|u\|_{0, \Omega}^{2}, \\
\mathcal{L}_{\Pi}(x, \lambda, \mu):= & \frac{1}{2}\left\|y-\Pi y_{d}\right\|_{0, \Omega}^{2}+\frac{\nu}{2}\|u\|_{0, \Omega}^{2} \\
& +a(y, p)-(u+\Pi f, p)_{0, \Omega}-\langle\xi, p\rangle_{-1,1, \Omega} \\
& -\langle\xi, \mu\rangle_{-1,1, \Omega}-\langle\lambda, y\rangle_{-1,1, \Omega} .
\end{aligned}
$$

At this point, we leave it open to choose $\Pi$. For instance, one may consider $\Pi$ either to be equal to the identity on $L^{2}(\Omega)$ or the $L^{2}$-projection onto $Y_{h}$. With this notation, we can formulate a discrete stationarity system which is independent of $\Pi$ because of the projection property (2.18). Concerning our subsequent error estimation, however, we are still flexible to estimate the error in the objective with respect to either projected (discrete) or continuous data; see Remark 3.5.

The discrete optimal control problem finally reads: for given data $f, y_{d}$ and $\nu>0$, find a solution $\left(y_{h}, u_{h}, \xi_{h}\right) \in$ $Y_{h} \times U_{h} \times \Xi_{h}$ to the problem

$$
\begin{array}{ll}
\text { Minimize } & J_{\Pi}\left(y_{h}, u_{h}\right) \\
\text { such that } & a\left(y_{h}, \phi_{h}\right)-\left(u_{h}+\Pi f, \phi_{h}\right)_{0, \Omega}-\left\langle\xi_{h}, \phi_{h}\right\rangle_{-1,1, \Omega}=0 \quad\left(\forall \phi_{h} \in Y_{h}\right), \\
& y_{h} \geq 0, \quad \xi_{h} \geq 0 \quad \text { and } \quad\left\langle\xi_{h}, y_{h}\right\rangle_{-1,1, \Omega}=0 .
\end{array}
$$

Utilizing standard techniques, one readily obtains the existence of a solution to (2.21). The vector representation of the latter satisfies the following discrete C-stationarity system (which does not depend on $\Pi$ owing to (2.18)) with multipliers $\mathbf{p}_{\mathbf{h}}, \boldsymbol{\lambda}_{\mathbf{h}}$ and $\boldsymbol{\mu}_{\mathbf{h}} \in \mathbb{R}^{\left|\mathcal{N}_{Y_{h}}\right|}$,

$$
\begin{aligned}
\mathcal{A}_{h} \mathbf{y}_{\mathbf{h}}-\mathcal{M}_{h} \mathbf{u}_{\mathbf{h}}-\boldsymbol{\xi}_{\mathbf{h}} & =\mathbf{f}_{\mathbf{h}}, \\
\boldsymbol{\xi}_{\mathbf{h}} \geq 0, \mathbf{y}_{\mathbf{h}} \geq 0, \mathbf{y}_{\mathbf{h}}{ }^{T} \boldsymbol{\xi}_{\mathbf{h}} & =0 \\
\mathcal{M}_{h}^{Y} \mathbf{y}_{\mathbf{h}}-\boldsymbol{\lambda}_{\mathbf{h}}+\mathcal{A}_{h} \mathbf{p}_{\mathbf{h}} & =\mathbf{y}_{\mathbf{d h}}, \\
\nu \mathcal{M}_{h}^{U} \mathbf{u}_{\mathbf{h}}-\mathcal{M}_{h} \mathbf{p}_{\mathbf{h}} & =0 \\
-\boldsymbol{\mu}_{\mathbf{h}}-\mathbf{p}_{\mathbf{h}} & =0 \\
\boldsymbol{\lambda}_{\mathbf{h}, z} & =0 \text { if } \mathbf{y}_{\mathbf{h}, z}>0, \quad \forall z \in \mathcal{N}_{Y_{h}}, \\
\boldsymbol{\mu}_{\mathbf{h}, z} & =0 \text { if } \boldsymbol{\xi}_{\mathbf{h}, z}>0, \quad \forall z \in \mathcal{N}_{Y_{h}}, \\
\boldsymbol{\lambda}_{\mathbf{h}}{ }^{T} \boldsymbol{\mu}_{\mathbf{h}} & \geq 0 .
\end{aligned}
$$

An optimal solution of (2.21) is strongly stationary, when it satisfies (2.22) and the following sign conditions on the biactive set,

$$
\boldsymbol{\mu}_{\mathbf{h}, z} \geq 0 \text { and } \boldsymbol{\lambda}_{\mathbf{h}, z} \geq 0, \quad \text { if } \quad \mathbf{y}_{\mathbf{h}, z}=0 \text { and } \boldsymbol{\xi}_{\mathbf{h}, z}=0 \quad \text { for } z \in \mathcal{N}_{Y_{h}}
$$


The multipliers $\mathbf{p}_{\mathbf{h}}$ and $\boldsymbol{\mu}_{\mathbf{h}}$ may be interpreted as coefficient vectors pertinent to functions in $Y_{h}$, whereas $\lambda_{\mathbf{h}}$ may be interpreted analogously to $\boldsymbol{\xi}_{\mathbf{h}}$. Therefore, with these multipliers, we associate a tuple of functions $\left(p_{h}, \lambda_{h}, \mu_{h}\right) \in Y_{h} \times \Xi_{h} \times Y_{h}$. For the ease of notation, we abbreviate $X_{h}=Y_{h} \times U_{h} \times \Xi_{h} \times Y_{h}$ and say that $\left(x_{h}^{\star}, \lambda_{h}^{\star}, \mu_{h}^{\star}\right) \in X_{h} \times \Xi_{h} \times Y_{h}$ is C- or strongly stationary, if its vector representation is C- or strongly stationary.

Remark 2.8. Suppose that $\left(y_{h}^{\star}, u_{h}^{\star}, \xi_{h}^{\star}\right)$ is C-stationary for $(2.21)$ with associated multipliers $\left(p_{h}^{\star}, \lambda_{h}^{\star}, \mu_{h}^{\star}\right) \in$ $Y_{h} \times \Xi_{h} \times Y_{h}$. Then we have

$$
\begin{aligned}
J\left(y_{h}^{\star}, u_{h}^{\star}\right) & =J_{\Pi}\left(y_{h}^{\star}, u_{h}^{\star}\right)+\frac{1}{2}\left(\left\|y_{d}\right\|_{0, \Omega}^{2}-\left\|\Pi y_{d}\right\|_{0, \Omega}^{2}\right) \\
& =\mathcal{L}_{\Pi}\left(x_{h}^{\star}, \lambda_{h}^{\star}, \mu_{h}^{\star}\right)+\frac{1}{2}\left(\left\|y_{d}\right\|_{0, \Omega}^{2}-\left\|\Pi y_{d}\right\|_{0, \Omega}^{2}\right) \\
& =\mathcal{L}\left(x_{h}^{\star}, \lambda_{h}^{\star}, \mu_{h}^{\star}\right),
\end{aligned}
$$

and for all $x_{h} \in X_{h}$,

$$
\nabla_{x} \mathcal{L}\left(x_{h}^{\star}, \lambda_{h}^{\star}, \mu_{h}^{\star}\right)\left(x_{h}\right)=\nabla_{x} \mathcal{L}_{\Pi}\left(x_{h}^{\star}, \lambda_{h}^{\star}, \mu_{h}^{\star}\right)\left(x_{h}\right)=0 .
$$

\section{Primal-Dual-Weighted error Representation}

In our numerics, the adaptive mesh refinement will be guided by the error with respect to the objective functional, but other choices such as linear or differentiable error functionals as in [8] are conceivable. This would, however, require a framework extending the one in [8] to non-smooth systems. Here, the objective represents the target quantity in our goal-oriented mesh adaption approach. For this purpose, we next establish the associated error representation. Our technique is related to the one in [16].

Theorem 3.1. Assume that $\left(y^{\star}, u^{\star}, \xi^{\star}\right)$ and $\left(y_{h}^{\star}, u_{h}^{\star}, \xi_{h}^{\star}\right)$ are C-stationary for (2.2) and (2.21) with associated multipliers $\left(p^{\star}, \lambda^{\star}, \mu^{\star}\right)$ and $\left(p_{h}^{\star}, \lambda_{h}^{\star}, \mu_{h}^{\star}\right)$. Then, it holds that

$$
\begin{aligned}
J\left(y_{h}^{\star}, u_{h}^{\star}\right)-J\left(y^{\star}, u^{\star}\right)= & \frac{1}{2} \nabla_{x x} \mathcal{L}\left(x_{h}^{\star}-x^{\star}, x_{h}^{\star}-x^{\star}\right) \\
& +\left\langle\lambda^{\star}, y_{h}^{\star}\right\rangle_{-1,1, \Omega}+\left\langle\xi_{h}^{\star}, \mu^{\star}\right\rangle_{-1,1, \Omega} .
\end{aligned}
$$

Proof. In view of (2.24), we have

$$
J\left(u_{h}^{\star}, y_{h}^{\star}\right)-J\left(u^{\star}, y^{\star}\right)=\mathcal{L}\left(x_{h}^{\star}, \lambda_{h}^{\star}, \mu_{h}^{\star}\right)-J\left(u^{\star}, y^{\star}\right) .
$$

Since the objective functional and the Lagrangian are quadratic, a Taylor expansion of $\mathcal{L}\left(x_{h}^{\star}, \lambda_{h}^{\star}, \mu_{h}^{\star}\right)$ at $x^{\star}$ yields

$$
\begin{aligned}
\mathcal{L}\left(x_{h}^{\star}, \lambda_{h}^{\star}, \mu_{h}^{\star}\right)= & \mathcal{L}\left(x^{\star}, \lambda_{h}^{\star}, \mu_{h}^{\star}\right)+\nabla_{x} \mathcal{L}\left(x^{\star}, \lambda_{h}^{\star}, \mu_{h}^{\star}\right)\left(x_{h}^{\star}-x^{\star}\right) \\
& +\frac{1}{2} \nabla_{x x} \mathcal{L}\left(x_{h}^{\star}-x^{\star}, x_{h}^{\star}-x^{\star}\right) .
\end{aligned}
$$

For the first term on the right-hand side in (3.3), we use the definition of $\mathcal{L}$ in (2.5) and the system (2.3) to obtain

$$
\mathcal{L}\left(x^{\star}, \lambda_{h}^{\star}, \mu_{h}^{\star}\right)=J\left(y^{\star}, u^{\star}\right)-\left\langle\lambda_{h}^{\star}, y^{\star}\right\rangle_{-1,1, \Omega}-\left\langle\xi^{\star}, \mu_{h}^{\star}\right\rangle_{-1,1, \Omega} .
$$

Taking again advantage of (2.3), for the second term on the right-hand side in (3.3) we find

$$
\begin{aligned}
\nabla_{x} \mathcal{L}\left(x^{\star}, \lambda_{h}^{\star}, \mu_{h}^{\star}\right)\left(x_{h}^{\star}-x^{\star}\right)= & \left\langle\lambda^{\star}, y_{h}^{\star}\right\rangle_{-1,1, \Omega}+\left\langle\lambda_{h}^{\star}, y^{\star}\right\rangle_{-1,1, \Omega} \\
& +\left\langle\xi_{h}^{\star}, \mu^{\star}\right\rangle_{-1,1, \Omega}+\left\langle\xi^{\star}, \mu_{h}^{\star}\right\rangle_{-1,1, \Omega} .
\end{aligned}
$$

Using (3.3)-(3.5) in (3.2) gives the assertion. 
The following lemma provides a representation of the second order Gâteaux derivative of the MPCCLagrangian.

Lemma 3.2. Under the same assumptions as in Theorem 3.1, for a tuple of discrete functions $\phi_{h}=$ $\left(\delta y_{h}, \delta u_{h}, \delta \xi_{h}, \delta p_{h}\right) \in X_{h}$ it holds that

$$
\begin{aligned}
\nabla_{x x} \mathcal{L}\left(x_{h}^{\star}-x^{\star}, x_{h}^{\star}-x^{\star}\right) & =\nabla_{x} \mathcal{L}\left(x_{h}^{\star}, \lambda_{h}^{\star}, \mu_{h}^{\star}\right)\left(x_{h}^{\star}-x^{\star}+\phi_{h}\right) \\
-\left\langle\xi_{h}^{\star}, \mu^{\star}\right\rangle_{-1,1, \Omega}- & \left\langle\xi^{\star}, \mu_{h}^{\star}\right\rangle_{-1,1, \Omega}-\left\langle\lambda^{\star}, y_{h}^{\star}\right\rangle_{-1,1, \Omega}-\left\langle\lambda_{h}^{\star}, y^{\star}\right\rangle_{-1,1, \Omega} .
\end{aligned}
$$

Proof. Taylor expansion of $\nabla_{x} \mathcal{L}\left(x_{h}^{\star}, \lambda_{h}^{\star}, \mu_{h}^{\star}\right)\left(x^{\star}-x_{h}^{\star}\right)$ at the point $x^{\star}$ and evaluation of $\nabla_{x} \mathcal{L}\left(x^{\star}, \lambda_{h}^{\star}, \mu_{h}^{\star}\right)\left(x^{\star}-x_{h}^{\star}\right)$ leads to

$$
\begin{gathered}
\nabla_{x} \mathcal{L}\left(x_{h}^{\star}, \lambda_{h}^{\star}, \mu_{h}^{\star}\right)\left(x_{h}^{\star}-x^{\star}\right)=\nabla_{x} \mathcal{L}\left(x^{\star}, \lambda_{h}^{\star}, \mu_{h}^{\star}\right)\left(x_{h}^{\star}-x^{\star}\right)+\nabla_{x x} \mathcal{L}\left(x_{h}^{\star}-x^{\star}, x_{h}^{\star}-x^{\star}\right) \\
=\left\langle\xi_{h}^{\star}, \mu^{\star}\right\rangle_{-1,1, \Omega}+\left\langle\xi^{\star}, \mu_{h}^{\star}\right\rangle_{-1,1, \Omega}+\left\langle\lambda^{\star}, y_{h}^{\star}\right\rangle_{-1,1, \Omega} \\
+\left\langle\lambda_{h}^{\star}, y^{\star}\right\rangle_{-1,1, \Omega}+\nabla_{x x} \mathcal{L}\left(x_{h}^{\star}-x^{\star}, x_{h}^{\star}-x^{\star}\right)
\end{gathered}
$$

which readily implies

$$
\begin{aligned}
\nabla_{x x} \mathcal{L}\left(x_{h}^{\star}-x^{\star}, x_{h}^{\star}-x^{\star}\right)= & \nabla_{x} \mathcal{L}\left(x_{h}^{\star}, \lambda_{h}^{\star}, \mu_{h}^{\star}\right)\left(x_{h}^{\star}-x^{\star}\right)-\left\langle\xi_{h}^{\star}, \mu^{\star}\right\rangle_{-1,1, \Omega} \\
& -\left\langle\xi^{\star}, \mu_{h}^{\star}\right\rangle_{-1,1, \Omega}-\left\langle\lambda^{\star}, y_{h}^{\star}\right\rangle_{-1,1, \Omega}-\left\langle\lambda_{h}^{\star}, y^{\star}\right\rangle_{-1,1, \Omega} .
\end{aligned}
$$

According to (2.25), it holds that $\nabla_{x} \mathcal{L}\left(x_{h}^{\star}, \lambda_{h}^{\star}, \mu_{h}^{\star}\right)\left(\phi_{h}\right)=0$. This yields the assertion.

In combination with Theorem 3.1, the previous lemma gives rise to an error representation which will be the basis for the evaluation of the primal-dual weighted residuals.

Theorem 3.3. In addition to the assumptions in Theorem 3.1 let $\delta x_{h}=\left(\delta y_{h}, \delta u_{h}, \delta \xi_{h}, \delta p_{h}\right) \in X_{h}$. Then, it holds that

$$
\begin{aligned}
J\left(u^{\star}, y^{\star}\right)-J\left(u_{h}^{\star}, y_{h}^{\star}\right)= & \frac{1}{2} a\left(y_{h}^{\star}, p^{\star}-\delta p_{h}\right)-\frac{1}{2}\left(u_{h}^{\star}+f, p^{\star}-\delta p_{h}\right)_{0, \Omega}-\frac{1}{2}\left\langle\xi_{h}^{\star}, p^{\star}-\delta p_{h}\right\rangle_{-1,1, \Omega} \\
& +\frac{1}{2} a\left(y^{\star}-\delta y_{h}, p_{h}^{\star}\right)+\frac{1}{2}\left(y_{h}^{\star}-y_{d}, y^{\star}-\delta y_{h}\right)_{0, \Omega}-\frac{1}{2}\left\langle\lambda_{h}^{\star}, y^{\star}-\delta y_{h}\right\rangle_{-1,1, \Omega} \\
& +\frac{1}{2}\left(\nu u_{h}^{\star}-p_{h}^{\star}, u^{\star}-\delta u_{h}\right)_{0, \Omega} \\
& +\frac{1}{2}\left\langle\xi^{\star}-\delta \xi_{h},-\mu_{h}^{\star}-p_{h}^{\star}\right\rangle_{-1,1, \Omega} \\
& -\frac{1}{2}\left\langle\lambda^{\star}, y_{h}^{\star}\right\rangle_{-1,1, \Omega}+\frac{1}{2}\left\langle\lambda_{h}^{\star}, y^{\star}\right\rangle_{-1,1, \Omega}-\frac{1}{2}\left\langle\xi_{h}^{\star}, \mu^{\star}\right\rangle_{-1,1, \Omega}+\frac{1}{2}\left\langle\xi^{\star}, \mu_{h}^{\star}\right\rangle_{-1,1, \Omega} .
\end{aligned}
$$

Proof. The combination of (3.1) from Theorem 3.1 and (3.6) from Lemma 3.2 reveals

$$
\begin{aligned}
J\left(u^{\star}, y^{\star}\right)-J\left(u_{h}^{\star}, y_{h}^{\star}\right)=-\frac{1}{2} \nabla_{x x} \mathcal{L}\left(x_{h}^{\star}-x^{\star}, x_{h}^{\star}-x^{\star}\right)-\left\langle\lambda^{\star}, y_{h}^{\star}\right\rangle_{-1,1, \Omega}-\left\langle\xi_{h}^{\star}, \mu^{\star}\right\rangle_{-1,1, \Omega} \\
=\frac{1}{2} \nabla_{x} \mathcal{L}\left(x_{h}^{\star}, \lambda_{h}^{\star}, \mu_{h}^{\star}\right)\left(x^{\star}-x_{h}^{\star}-\phi_{h}\right)-\frac{1}{2}\left\langle\xi_{h}^{\star}, \mu^{\star}\right\rangle_{-1,1, \Omega}+\frac{1}{2}\left\langle\xi^{\star}, \mu_{h}^{\star}\right\rangle_{-1,1, \Omega} \\
\\
\quad-\frac{1}{2}\left\langle\lambda^{\star}, y_{h}^{\star}\right\rangle_{-1,1, \Omega}+\frac{1}{2}\left\langle\lambda_{h}^{\star}, y^{\star}\right\rangle_{-1,1, \Omega} .
\end{aligned}
$$

Choosing $\phi_{h}=\delta x_{h}-x_{h}^{\star}=\left(\delta y_{h}-y_{h}^{\star}, \delta u_{h}-u_{h}^{\star}, \delta \xi_{h}-\xi_{h}^{\star}, \delta p_{h}-p_{h}^{\star}\right)$ and exploiting the structure of the MPCC-Lagrangian provides the assertion. 
Remark 3.4. The terms (3.8a) - (3.8d) in Theorem 3.3 represent the dual and the primal error in the feasibility and optimality conditions $(2.22 \mathrm{a}),(2.22 \mathrm{c}),(2.22 \mathrm{~d}),(2.22 \mathrm{e})$, whereas the remaining terms $(3.8 \mathrm{e})$ reflect the mismatch in complementarity as expressed by $(2.22 \mathrm{f})$ and $(2.22 \mathrm{~g})$. We further note that the sign condition on $\langle\lambda, \mu\rangle_{-1,1, \Omega}$ is not reflected in the representation found in Theorem 3.3. This can be attributed to the facts that $\langle\lambda, \mu\rangle_{-1,1, \Omega}$ involves dual quantities only and that this pairing is of course not part of the MPCC-Lagrangian. This is a consequence of the special nature of MPCCs, which violate standard constraint qualifications for the existence (and boundedness) of Lagrange multipliers.

Remark 3.5. Note that in Theorems 3.1 and 3.3 one may alternatively consider the error $J_{\Pi}\left(y_{h}^{\star}, u_{h}^{\star}\right)-J\left(y^{\star}, u^{\star}\right)$. In this situation, the error representation includes additional data terms pertinent to replacing $y_{d}$ by $\Pi y_{d}$ in the objective, i.e.,

$$
J_{\Pi}\left(y_{h}^{\star}, u_{h}^{\star}\right)-J\left(y^{\star}, u^{\star}\right)=J\left(y_{h}^{\star}, u_{h}^{\star}\right)-J\left(y^{\star}, u^{\star}\right)+\frac{1}{2}\left\|\Pi y_{d}\right\|_{0, \Omega}^{2}-\frac{1}{2}\left\|y_{d}\right\|_{0, \Omega}^{2} .
$$

\section{Primal-Dual-Weighted a POSteriori estimator}

Note that the representation of $J\left(y^{\star}, u^{\star}\right)-J_{\Pi}\left(y_{h}^{\star}, u_{h}^{\star}\right)$ is not fully a posteriori, which is, on the one hand, due to the weights $x^{\star}-\delta x_{h}$ and, on the other hand, due to the dependence of the terms (3.8e) on $\lambda^{\star}, y^{\star}, \mu^{\star}$ and $\xi^{\star}$. This fact prevents an immediate numerical realization of the representation in Theorem 3.3. In this section we deduce a fully a posteriori and local estimator from the terms in the error representation in Theorem 3.3. Below, we utilize a specific discretization based on simplices and $P_{1,0}$-elements. We emphasize, however, that other discretizations are possible; see, e.g., [8].

\subsection{Detailed discretization}

Subsequently, we exemplarily consider specific choices for the bilinear form $a$ and the discrete spaces. In fact, we set $a(y, z):=\int_{\Omega} \nabla y \cdot \nabla z \mathrm{~d} x$ on $H_{0}^{1}(\Omega) \times H_{0}^{1}(\Omega)$. Our numerical tests in Section 5.3 all have $\Omega \subset \mathbb{R}^{2}$. For this reason and without loss of generality, we restrict ourselves from now on to polygonal, bounded domains $\Omega \subset \mathbb{R}^{2}$. Further let $\mathcal{T}$ be a triangulation of $\Omega$ (into triangles) in the sense of ([1], p. 394). The set of all edges of triangles in $\mathcal{T}$ is denoted by $\mathcal{E}$, whereas $\mathcal{N}$ is the set of nodes of triangles in $\mathcal{T}$. For a subset $\omega \subset \Omega$, we write $\mathcal{T}(\omega):=\{T \in \mathcal{T} \mid T \subset \omega\}$, and, analogously, $\mathcal{E}(\omega):=\{E \in \mathcal{E} \mid E \subset \omega\}$ and $\mathcal{N}(\omega):=\{z \in \mathcal{N} \mid z \in \omega\}$.

By $P_{1,0}(\mathcal{T})$ we denote the space of $\mathcal{T}$-piecewise affine and globally continuous functions on $\Omega$ with zero boundary conditions and the usual nodal basis

$$
\left\{\varphi_{z} \in P_{1,0}(\mathcal{T}) \mid z \in \mathcal{N}, \forall \bar{z} \in \mathcal{N}, \varphi_{z}(\bar{z})=\delta_{z, \bar{z}}\right\}
$$

where $\delta_{z, \bar{z}}$ denotes the Kronecker-Delta with $\delta_{z, \bar{z}}=1$ if $z=\bar{z}$ and $\delta_{z, \bar{z}}=0$ otherwise. We set $Y_{h}=P_{1,0}(\mathcal{T})$ as well as $U_{h}=P_{1,0}(\mathcal{T})$. Then, by $(2.22 \mathrm{~d})$ and $(2.22 \mathrm{e})$, the terms $(3.8 \mathrm{c})$ and $(3.8 \mathrm{~d})$ in the difference of the objectives yield zero. Indeed, $M_{h}^{Y}=M_{h}$ is an invertible matrix, and thus $p_{h}^{\star}=\nu u_{h}^{\star}$ and $\mu_{h}^{\star}=-p_{h}^{\star}$.

On every triangle $T \in \mathcal{T}$ we approximate $\left.y^{\star}\right|_{T}$ (and $\left.p^{\star}\right|_{T}$ ) by the quadratic interpolant $\tilde{y}_{h}^{\star}$ of $y_{h}^{\star}$ based on the (at most) six nodes of $\bigcup\{S \in \mathcal{T} \mid S \cap T \in \mathcal{T} \cup \mathcal{E}\}$ and $\tilde{p}_{h}^{\star}$ of $p_{h}^{\star}$, respectively (cf. [8]). The left hand side in Figure 1 shows the situation in the interior of the domain, whereas the right hand side shows a triangle at the boundary of the domain (ruled), where the boundary edge has no neighboring node outside of the triangle. To overcome this, we enlarge the triangulation by outer parallelogram points as depicted in Figure 1.

We define the edge jumps of a discrete function $v_{h}=\phi_{h} \in Y_{h}$ with the $\mathcal{T}$-piecewise constant gradient $\nabla v_{h}$ as follows: for a triangle $T \in \mathcal{T}$ with an edge $E \in \mathcal{E}(T)$ we denote by $\nu_{T, E}$ the outer unit normal vector of $T$ at the edge $E$. Assuming that $E=T_{+} \cap T_{-}$, we note that $\nu_{T_{-}, E}=-\nu_{T_{+}, E}$ and thus, the edge jump

$$
\left[\nabla v_{h}\right]_{E}:=\left(\left.\nabla v_{h}\right|_{T_{+}}-\left.\nabla v_{h}\right|_{T_{-}}\right) \cdot \nu_{T_{+}, E}=\left(\left.\nabla v_{h}\right|_{T_{-}}-\left.\nabla v_{h}\right|_{T_{+}}\right) \cdot \nu_{T_{-}, E}
$$



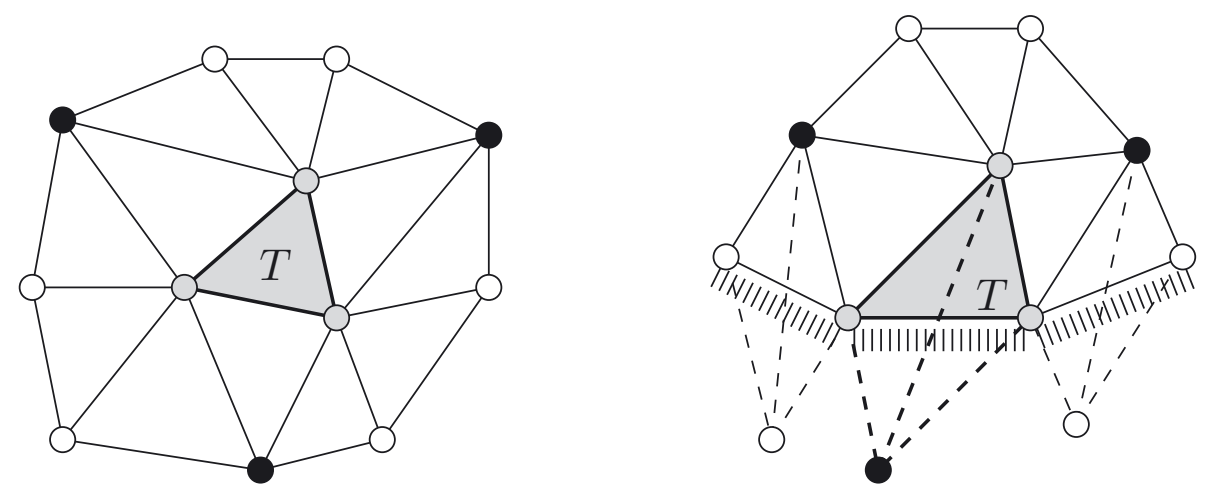

FiguRE 1. The $P_{2}$ interpolation of discrete functions given in the nodes of the triangulation on one triangle $T$ (gray shaded) is defined via its values in the nodes of $T$ (gray circles) and of the (up to) three triangles sharing an edge with $T$ (black circles). To overcome the lack of neighboring nodes at the boundary, we enlarge the triangulation as depicted on the right hand side.

is independent of the permutation of $T_{+}$and $T_{-}$. If $E \subset \partial \Omega$, we define $\left[\nabla v_{h}\right]_{E}:=0$. For $z \in H_{0}^{1}(\Omega), \mathcal{T}$-piecewise integration by parts yields

$$
a\left(\phi_{h}, z\right)=\sum_{T \in \mathcal{T}} \sum_{E \in \mathcal{E}(T \backslash \partial \Omega)} \nabla \phi_{h} \cdot \nu_{T, E} \int_{E} z \mathrm{~d} S=\sum_{E \in \mathcal{E}}\left[\nabla \phi_{h}\right]_{E} \int_{E} z \mathrm{~d} S .
$$

Rearranging the edge terms according to triangles we obtain the identity

$$
a\left(\phi_{h}, z\right)=\sum_{T \in \mathcal{T}} \sum_{E \in \mathcal{E}(T)} \frac{1}{2}\left[\nabla \phi_{h}\right]_{E} \int_{E} z \mathrm{~d} S=\sum_{T \in \mathcal{T}} \frac{1}{2}\left[\nabla \phi_{h}\right]_{\partial T} \int_{\partial T} z \mathrm{~d} S .
$$

The total error estimator, which we derive below, has the structure

$$
\eta_{h}:=\sum_{T \in \mathcal{T}} \eta_{T}
$$

where $\eta_{T}=\eta_{\mathrm{PDE} 1, \mathrm{~T}}+\eta_{\mathrm{PDE} 2, \mathrm{~T}}+\eta_{\mathrm{CM}, \mathrm{T}}$. On every triangle $T, \eta_{\mathrm{PDE} 1, \mathrm{~T}}$ reflects the error contribution of the dualweighted primal residual (3.8a) on $T$, while $\eta_{\mathrm{PDE} 2 \mathrm{~T}}$ contains the error contribution of the primal-weighted dual equation (3.8b) on $T$. These estimators are derived in Section 4.2. The complementarity mismatch contribution to the error is contained in $\eta_{\mathrm{CM}, \mathrm{T}}$, which is discussed in Section 4.3.

\subsection{Primal-dual-weighted PDE residuals}

For $\delta p_{h} \in P_{1,0}(\mathcal{T})$, we use (4.1) and the $L^{2}$-representation of $\boldsymbol{\xi}_{\mathbf{h}}$ due to (2.16) to write (3.8a) in Theorem 3.3 as

$$
\begin{aligned}
& a\left(y_{h}^{\star}, p^{\star}-\delta p_{h}\right)-\int_{T}\left(u_{h}^{\star}+f\right)\left(p^{\star}-\delta p_{h}\right) \mathrm{d} x-\left\langle\xi_{h}^{\star}, p^{\star}-\delta p_{h}\right\rangle_{-1,1, \Omega} \\
= & \sum_{T \in \mathcal{T}}\left(\sum_{E \in \mathcal{E}(T)}\left(\frac{1}{2}\left[\nabla y_{h}\right]_{E} \int_{E} p^{\star}-\delta p_{h} \mathrm{~d} S\right)-\int_{T}\left(u_{h}^{\star}+f+\xi_{h}^{\star}\right)\left(p^{\star}-\delta p_{h}\right) \mathrm{d} x\right) .
\end{aligned}
$$

We set $\delta p_{h}=\nu u_{h}^{\star}$, estimate $p^{\star}$ by $\nu \tilde{u}_{h}^{\star}$ (cf. above section) and recall (2.17) to obtain from (4.3)

$$
\eta_{\mathrm{PDE} 1, \mathrm{~T}}:=\frac{1}{2}\left|-\int_{T} \nu\left(u_{h}^{\star}+f+\xi_{h}^{\star}\right)\left(\tilde{u}_{h}^{\star}-u_{h}^{\star}\right) \mathrm{d} x+\int_{\partial T} \frac{\nu}{2}\left[\nabla y_{h}^{\star}\right]_{\partial T}\left(\tilde{u}_{h}^{\star}-u_{h}^{\star}\right) \mathrm{d} S\right| .
$$


An analogue procedure applies to the weighted residual of the adjoint equation (3.8b), and we obtain

$$
\eta_{\mathrm{PDE} 2, \mathrm{~T}}:=\frac{1}{2}\left|\int_{T}\left(y_{h}^{\star}-y_{d}-\lambda_{h}^{\star}\right)\left(\tilde{y}_{h}^{\star}-y_{h}^{\star}\right) \mathrm{d} x+\int_{\partial T} \frac{\nu}{2}\left[\nabla u_{h}^{\star}\right]_{\partial T}\left(\tilde{y}_{h}^{\star}-y_{h}^{\star}\right) \mathrm{d} S\right| .
$$

As a consequence, the terms in

$$
\eta_{\mathrm{PDE} 1}:=\sum_{T \in \mathcal{T}} \eta_{\mathrm{PDE} 1, \mathrm{~T}}, \quad \quad \eta_{\mathrm{PDE} 2}:=\sum_{T \in \mathcal{T}} \eta_{\mathrm{PDE} 2, \mathrm{~T}}
$$

are fully a posteriori. The integrals can be evaluated by Gauss quadrature rules which are exact for polynomials up to a certain degree.

\subsection{Complementarity mismatch}

The weighted complementarity residual is given by the sum

$$
\frac{1}{2}\left(\left\langle\xi^{\star}, \mu_{h}^{\star}\right\rangle_{-1,1, \Omega}-\left\langle\xi_{h}^{\star}, \mu^{\star}\right\rangle_{-1,1, \Omega}+\left\langle\lambda_{h}^{\star}, y^{\star}\right\rangle_{-1,1, \Omega}-\left\langle\lambda^{\star}, y_{h}^{\star}\right\rangle_{-1,1, \Omega}\right),
$$

and is next analyzed term by term. The resulting estimator is the sum of the a posteriori quantities defined in $(4.6)-(4.9)$.

We begin our analysis with the terms that can be understood as $L^{2}$-products due to (2.16). In fact, considering $\left\langle\lambda_{h}^{\star}, y^{\star}\right\rangle_{-1,1, \Omega}=\left(\lambda_{h}^{\star}, y^{\star}\right)_{0, \Omega}$, inserting $\left(\lambda_{h}^{\star}, y_{h}^{\star}\right)_{0, \Omega}$ and replacing $y^{\star}$ by $\tilde{y}_{h}^{\star}$ we obtain the a posteriori estimator

$$
\eta_{\mathrm{CM} 1, \mathrm{~T}}:=\frac{1}{2}\left|\int_{T}\left(\tilde{y}_{h}^{\star}-y_{h}^{\star}\right) \lambda_{h}^{\star} \mathrm{d} x\right| .
$$

Further, subtracting $\left(\xi_{h}^{\star}, \nu u_{h}^{\star}\right)_{0, \Omega}$ from $-\left\langle\xi_{h}^{\star}, \mu^{\star}\right\rangle_{-1,1, \Omega}$ and replacing $\mu^{\star}$ by $-\nu \tilde{u}_{h}^{\star}$ we find

$$
\eta_{\mathrm{CM} 2, \mathrm{~T}}:=\frac{1}{2}\left|\int_{T} \nu\left(\tilde{u}_{h}^{\star}-u_{h}^{\star}\right) \xi_{h}^{\star} \mathrm{d} x\right| .
$$

Next, the term $\left\langle\xi^{\star}, \mu_{h}^{\star}\right\rangle_{-1,1, \Omega}$ which belongs to the mismatch of the continuous and discrete strongly active sets is analyzed. Due to $\left\langle\xi^{\star}, \mu^{\star}\right\rangle_{-1,1, \Omega}=0$ following from (2.3g), it holds that

$$
\left\langle\xi^{\star}, \mu_{h}^{\star}\right\rangle_{-1,1, \Omega}=\left\langle\xi^{\star}, \mu_{h}^{\star}-\mu^{\star}\right\rangle_{-1,1, \Omega} .
$$

In the same way as, e.g., in [34], equation (3.3), we exploit (2.3a) to conclude

$$
\begin{aligned}
\left\langle\xi^{\star}, \mu_{h}^{\star}-\mu^{\star}\right\rangle_{-1,1, \Omega} & =\left\langle\mathcal{A} y^{\star}-u^{\star}-f, \mu_{h}^{\star}-\mu^{\star}\right\rangle_{-1,1, \Omega} \\
& =\left(-u^{\star}-f, \mu_{h}^{\star}-\mu^{\star}\right)_{0, \Omega}+\left(\nabla y^{\star}, \nabla\left(\mu_{h}^{\star}-\mu^{\star}\right)\right)_{0, \Omega} .
\end{aligned}
$$

Similarly to above, we use $\mu^{\star}=-\nu u^{\star}$ and $\mu_{h}^{\star}=-\nu u_{h}^{\star}$ and replace $u^{\star}$ by $\tilde{u}_{h}^{\star}$ whenever it appears in the weight and by $u_{h}^{\star}$ in the residual, as well as we use $y_{h}^{\star}$ instead of $y^{\star}$. For $T \in \mathcal{T}$ we obtain from (4.1)

$$
\eta_{\mathrm{CM} 3, \mathrm{~T}}:=\frac{\nu}{2}\left|-\int_{T}\left(u_{h}^{\star}+f\right)\left(\tilde{u}_{h}^{\star}-u_{h}^{\star}\right) \mathrm{d} x+\int_{\partial T} \frac{1}{2}\left[\nabla y_{h}^{\star}\right]_{\partial T}\left(\tilde{u}_{h}^{\star}-u_{h}^{\star}\right) \mathrm{d} S\right| .
$$

Finally, for the term $\left\langle\lambda^{\star}, y_{h}^{\star}\right\rangle_{-1,1, \Omega}$, by $(2.3 \mathrm{c})$ it holds that

$$
\begin{aligned}
-\left\langle\lambda^{\star}, y_{h}^{\star}\right\rangle_{-1,1, \Omega} & =\left\langle y^{\star}+\mathcal{A}^{*} p^{\star}-y_{d}, y^{\star}-y_{h}^{\star}\right\rangle_{-1,1, \Omega} \\
& =\left(y^{\star}-y_{d}, y^{\star}-y_{h}^{\star}\right)_{0, \Omega}+\left(\nabla p^{\star}, \nabla\left(y^{\star}-y_{h}^{\star}\right)\right)_{0, \Omega} .
\end{aligned}
$$

We replace $y^{\star}$ by $\tilde{y}_{h}^{\star}$ when it appears in the weight and by $y_{h}^{\star}$ in the residual, and estimate

$$
\eta_{\mathrm{CM} 4 \mathrm{~T}}:=\frac{1}{2}\left|\int_{T}\left(y_{h}^{\star}-y_{d}\right)\left(\tilde{y}_{h}^{\star}-y_{h}^{\star}\right) \mathrm{d} x+\int_{\partial T} \frac{\nu}{2}\left[\nabla u_{h}^{\star}\right]_{\partial T}\left(\tilde{y}_{h}^{\star}-y_{h}^{\star}\right) \mathrm{d} S\right|,
$$

where we have used integration by parts as in (4.1). 


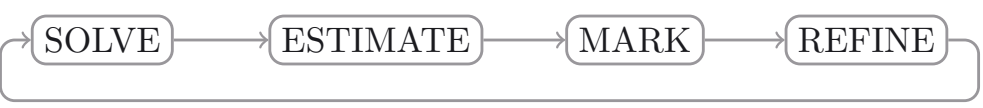

Figure 2. The typical adaptive scheme as described in Section 5.1.

\subsection{Total error}

Summarizing our above findings, we obtain the following estimator.

Let $\left(x^{\star}, \lambda^{\star}, \mu^{\star}\right) \in X \times H^{-1}(\Omega) \times H_{0}^{1}(\Omega)$ be a solution to problem $(2.2)$ and let $\left(x_{h}^{\star}, \lambda_{h}^{\star}, \mu_{h}^{\star}\right) \in X_{h} \times \Xi_{h} \times Y_{h}$ satisfy the conditions (2.22). With $\eta_{\mathrm{PDE} 1, \mathrm{~T}}$ from (4.4), $\eta_{\mathrm{PDE} 2, \mathrm{~T}}$ from (4.5) and $\eta_{\mathrm{CM}, \mathrm{T}}=\eta_{\mathrm{CM} 1, \mathrm{~T}}+\eta_{\mathrm{CM} 2, \mathrm{~T}}+$ $\eta_{\mathrm{CM} 3, \mathrm{~T}}+\eta_{\mathrm{CM} 4 \mathrm{~T}}$ from (4.6)-(4.9), we estimate the local distribution of the difference $J\left(u^{\star}, y^{\star}\right)-J\left(u_{h}^{\star}, y_{h}^{\star}\right)$ by

$$
\eta_{h}=\sum_{T \in \mathcal{T}} \eta_{\mathrm{PDE} 1, \mathrm{~T}}+\eta_{\mathrm{PDE} 2, \mathrm{~T}}+\eta_{\mathrm{CM}, \mathrm{T}}
$$

\section{NumERICS}

We start by a brief overview of the adaptive finite element method (AFEM) and its subroutines in Section 5.1 before establishing the optimization algorithm yielding a C-stationary point of problem (2.21) on every adaptive refinement level in Section 5.2. Finally, Section 5.3 contains numerical results.

\subsection{Adaptive scheme}

AFEM typically follows the scheme displayed in Figure 2. In this context, the mesh-adaption process is guided iteratively by local error indicators relying on solutions of the considered problem on the current mesh. Elements with large local error indicators are marked for refinement, and a superset of the marked elements is actually refined to maintain a regular mesh. The overall Algorithm 1 repeats this cycle until a given complexity $N$ (e.g. the number of degrees of freedom in the SOLVE procedure) is reached.

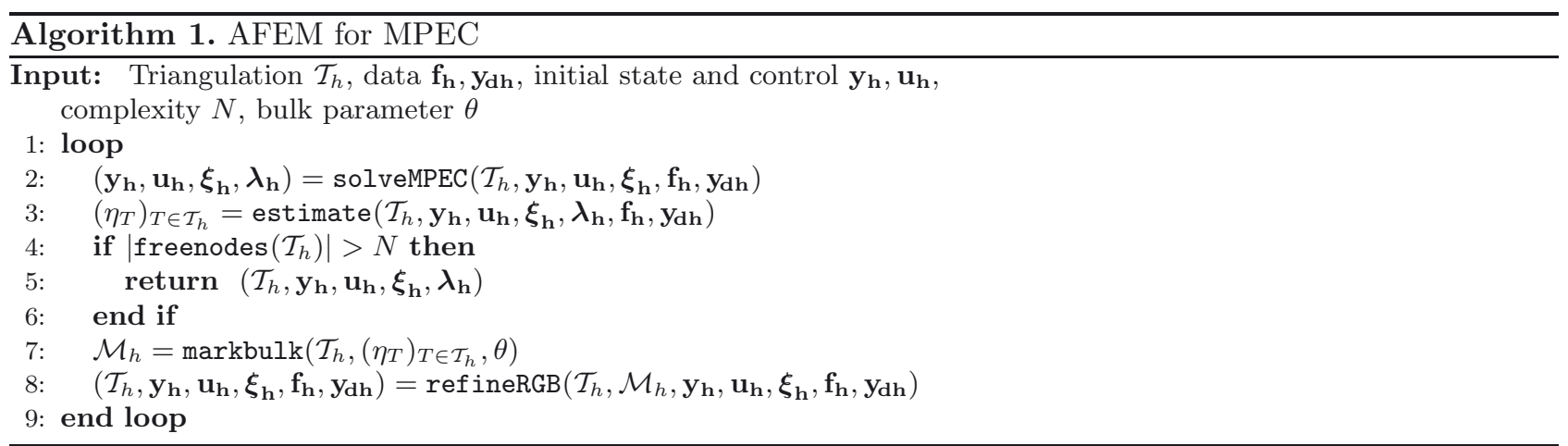

We briefly discuss the respective steps of Algorithm 1. The solution Step 2 (subroutine solveMPEC) is described in detail in Section 5.2. The calculation of a local error indicator

$$
\eta_{h}=\sum_{T \in \mathcal{T}} \eta_{T}
$$

from the discrete solutions on the coarse mesh $\mathcal{T}$ in Step 3 (subroutine estimate) follows from Section 4 . We use a bulk criterion (subroutine markbulk) to mark those elements in $\mathcal{T}$, which are refined to obtain the next 
AFEM level. In Step 7 this yields the set $\mathcal{M} \subset \mathcal{T}$ of minimal cardinality $|\mathcal{M}|$ such that for a bulk parameter $\theta \in(0,1)$ it holds that

$$
\theta \eta_{h} \leq \sum_{T \in \mathcal{M}} \eta_{T}
$$

This criterion stops the marking procedure, relying on a greedy algorithm, as soon as the total error on $\mathcal{M}$ has reached a fraction of $\theta$ of the total error on $\mathcal{T}$.

In the refinement Step 8 (subroutine refineRGB) one has to avoid hanging nodes and guarantee shape regularity of the triangulation. This is achieved by applying an algorithm, which marks additional triangles or edges; see also [12] or [3] for a similar, earlier treatment.

The AFEM level is indicated by $h$. In the adaptive scheme the maximum (local) mesh size is not required to converge to zero globally, as triangles may no longer get refined from some level $h$ on due to the error indicator.

\subsection{Optimization algorithm}

The discrete optimality system $(2.22)$ is solved iteratively through a sequence of penalized problems $\left(\mathrm{P}_{\gamma}\right)$. This strategy is inspired by [2]. Recalling the mass and stiffness matrices of Section 2.4, on a fixed AFEM level $h$ and for a penalty parameter $\gamma>0$ the penalized problem $\left(\mathrm{P}_{\gamma}\right)$ associated with $(2.21)$ reads

$$
\begin{array}{cl}
\text { Minimize } & \frac{1}{2}\left(\mathbf{y}-\mathcal{M}_{h}^{-1} \mathbf{y}_{\mathbf{d h}}\right)^{T} \mathcal{M}_{h}\left(\mathbf{y}-\mathcal{M}_{h}^{-1} \mathbf{y}_{\mathbf{d h}}\right)+\frac{\nu}{2} \mathbf{u}^{T} \mathcal{M}_{h} \mathbf{u}+\gamma \mathbf{y}^{T} \boldsymbol{\xi} \\
\text { such that } & \mathcal{A}_{h} \mathbf{y}=\mathcal{M}_{h} \mathbf{u}+\mathbf{f}_{\mathbf{h}}+\boldsymbol{\xi}, \\
\mathbf{y} & \geq 0 \text { and } \boldsymbol{\xi} \geq 0 .
\end{array}
$$

Note that a feasible point $(\mathbf{y}, \mathbf{u}, \boldsymbol{\xi})$ of $\left(\mathrm{P}_{\gamma}\right)$ satisfies $\mathbf{y}^{T} \boldsymbol{\xi} \geq 0$. Hence, $\left(\mathrm{P}_{\gamma}\right)$ penalizes violations of $\mathbf{y}^{T} \boldsymbol{\xi}=0$ by $\gamma>0$. The necessary first order optimality system of $\left(\mathrm{P}_{\gamma}\right)$ is given by

$$
\begin{array}{r}
\mathcal{R}_{\gamma, 1}:=\mathcal{M}_{h} \mathbf{y}+\gamma \boldsymbol{\xi}+\mathcal{A}_{h} \mathbf{p}-\boldsymbol{\lambda}-\mathbf{y}_{\mathbf{d h}}=0, \\
\nu \mathcal{M}_{h} \mathbf{u}-\mathcal{M}_{h} \mathbf{p}=0, \\
\gamma \mathbf{y}-\mathbf{p}-\boldsymbol{\mu}=0, \\
\mathcal{R}_{\gamma, 2}:=\mathcal{A}_{h} \mathbf{y}-\boldsymbol{\xi}-\mathcal{M}_{h} \mathbf{u}-\mathbf{f}_{\mathbf{h}}=0,
\end{array}
$$

and the complementarity conditions

$$
\begin{array}{ll}
\boldsymbol{\lambda} \geq 0, & \mathbf{y} \geq 0, \quad \boldsymbol{\lambda} \cdot \mathbf{y}=0 \\
\boldsymbol{\mu} \geq 0, & \boldsymbol{\xi} \geq 0, \quad \boldsymbol{\mu} \cdot \boldsymbol{\xi}=0 .
\end{array}
$$

We reduce the above system by eliminating the discrete adjoint state $\mathbf{p}$ and the multiplier $\boldsymbol{\mu}$. Using the $\max \{0, \cdot\}$ operator (componentwise) and an arbitrary, but fixed constant $c>0$, we reformulate (5.1e)-(5.1f) as

$$
\begin{aligned}
& \mathcal{R}_{\gamma, 3}:=\boldsymbol{\lambda}-\max \{0, \boldsymbol{\lambda}-c \mathbf{y}\}=0, \\
& \mathcal{R}_{\gamma, 4}:=\boldsymbol{\mu}-\max \{0, \boldsymbol{\mu}-c \boldsymbol{\xi}\}=0 .
\end{aligned}
$$

The resulting system (5.1a), (5.1d), (5.2) is solved by a semi-smooth Newton-method (Algorithm 2); see [20] for details on the latter. As an attempt towards globalization of the Newton solver, we employ a residual based strategy which adjusts the step size $\tau \in(0,1)$ in Algorithm 2 such that the total residual of system (5.1) decreases in each iteration. In this context, for $\mathbf{X}=(\mathbf{y}, \mathbf{u}, \boldsymbol{\xi}, \boldsymbol{\lambda})$, the residual residual $\mathbf{l}_{\gamma}(\mathbf{X})$ in Step 7 is the sum 
of four parts belonging to the four equations (5.1a), (5.1d), (5.2), that is,

$$
\begin{aligned}
\operatorname{res}_{\gamma, 1} & =\left|\mathcal{R}_{\gamma, 1}^{T} \mathcal{A}_{h}^{-1} \mathcal{R}_{\gamma, 1}\right|^{\frac{1}{2}} \\
\operatorname{res}_{\gamma, 2} & =\left|\mathcal{R}_{\gamma, 2}^{T} \mathcal{A}_{h}^{-1} \mathcal{R}_{\gamma, 2}\right|^{\frac{1}{2}} \\
\operatorname{res}_{\gamma, 3} & =\left|\mathcal{R}_{\gamma, 3}\right|_{1} \\
\operatorname{res}_{\gamma, 4} & =\left|\mathcal{R}_{\gamma, 4}\right|_{1}, \\
\operatorname{residual}_{\gamma}(\mathbf{X}) & =\operatorname{res}_{\gamma, 1}+\operatorname{res}_{\gamma, 2}+\operatorname{res}_{\gamma, 3}+\operatorname{res}_{\gamma, 4} .
\end{aligned}
$$

In the associated Step 7 of Algorithm 2, we choose $\tau_{0}=\Sigma_{M}$, the machine precision. Note that our globalization strategy is a simple Armijo-type backtracking which worked well in our numerical practice. For theoretical investigations and guaranteed convergence, however, a more complex strategy like a path search $[13,36]$ would be necessary.

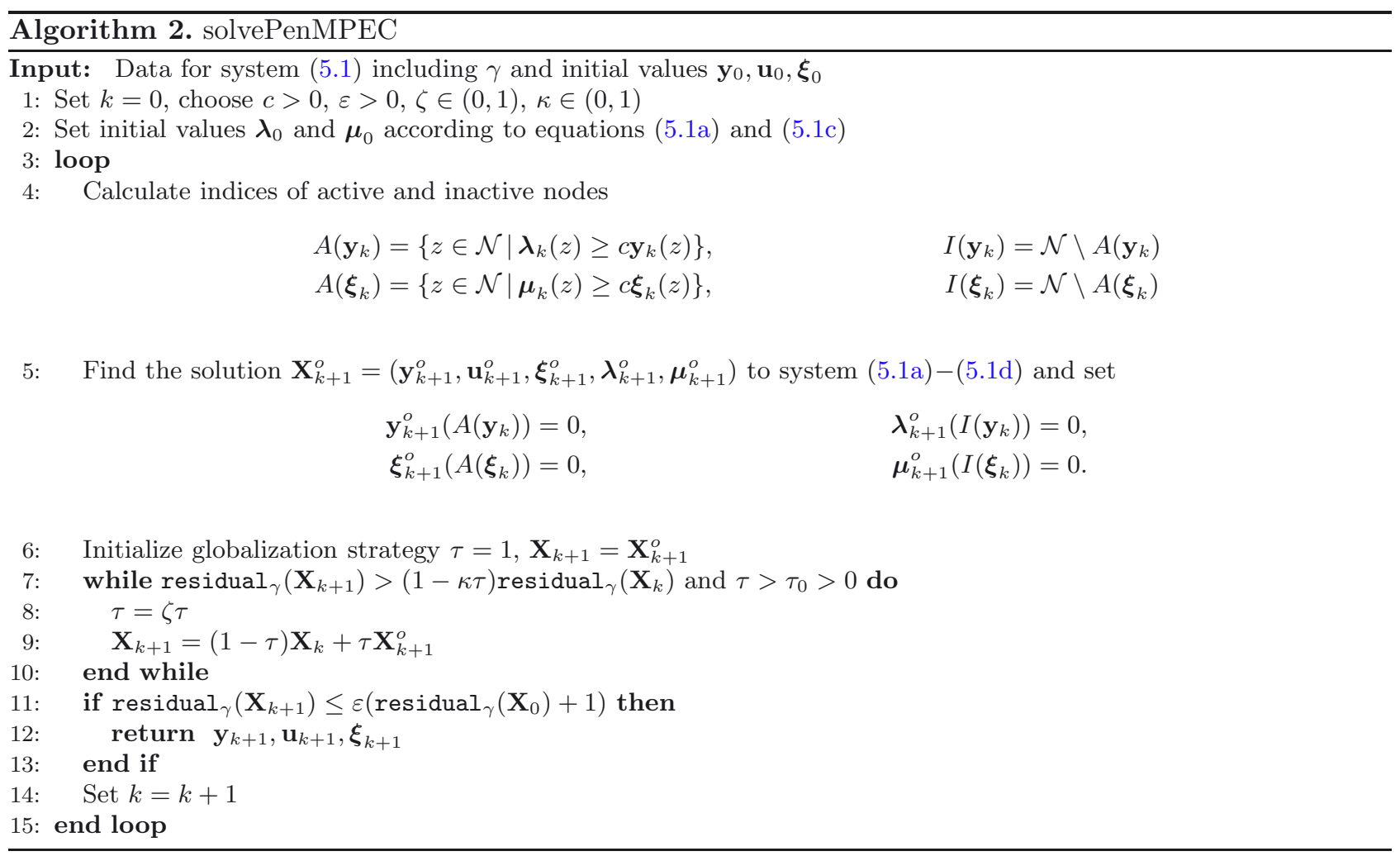

Algorithm 3 corresponds to Step 2 of Algorithm 1. Concerning its convergence in finite dimensional spaces, we observe that MPEC-LICQ (see [2], Def. 2) is generically satisfied in the problem class under consideration. Thus, due to [2], accumulation points of a series $\left(y_{\gamma}, u_{\gamma}, \xi_{\gamma}\right)_{\gamma}$ of solutions to the respective penalized problems for nondecreasing $\gamma$ are either not feasible for problem 2.2, or satisfy C-stationarity. For completeness we also mention that under additional assumptions one may even get a strongly stationary point. 
The residual in Step 5 of Algorithm 3 is computed as follows:

$$
\begin{array}{rlrl}
\mathcal{R}_{1} & :=\mathcal{A}_{h} \mathbf{y}-\mathcal{M}_{h} \mathbf{u}-\boldsymbol{\xi}-\mathbf{f}_{\mathbf{h}}, & \operatorname{res}_{1} & =\left(\mathcal{R}_{1}^{T} \mathcal{A}_{h}^{-1} \mathcal{R}_{1}\right)^{\frac{1}{2}} \\
\mathcal{R}_{2} & :=\mathcal{M}_{h} \mathbf{y}-\boldsymbol{\lambda}+\nu \mathcal{A}_{h} \mathbf{u}-\mathbf{y} \mathbf{d h}, & \operatorname{res}_{2}=\left(\mathcal{R}_{2} \mathcal{A}_{h}^{-1} \mathcal{R}_{2}\right)^{\frac{1}{2}} \\
\operatorname{res}_{3} & =|\mathbf{u} \boldsymbol{\xi}|_{1}, \quad \operatorname{res}_{4}=|\boldsymbol{\xi}-\max \{0, \boldsymbol{\xi}-c \mathbf{y}\}|_{1}, & \operatorname{res}_{5}=|\mathbf{y} \boldsymbol{\lambda}|_{1}, \\
& \text { residual }(\mathbf{y}, \mathbf{u}, \boldsymbol{\xi}, \boldsymbol{\lambda})=\operatorname{res}_{1}+\operatorname{res}_{2}+\operatorname{res}_{3}+\operatorname{res}_{4}+\operatorname{res}_{5} .
\end{array}
$$

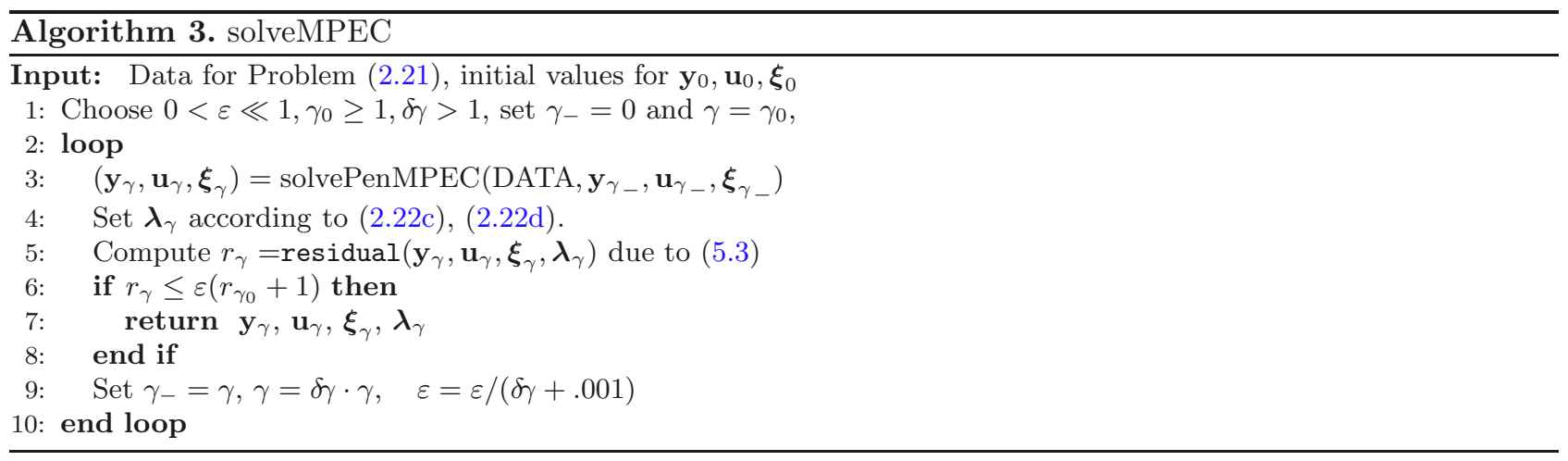

\subsection{Numerical results}

Finally, we present numerical results obtained by our AFEM approach. The bulk parameter in Algorithm 1 is set to $\theta=0.5$ in both examples.

Example 5.1. We tested our algorithm for solving ([21], Example 6.1), where $\mathcal{A}=-\Delta$ on the square domain $\Omega=(0,1) \times(0,1)$ and

$$
\begin{aligned}
z_{1}\left(x_{1}\right) & =-4096 x_{1}^{6}+6144 x_{1}^{5}-3072 x_{1}^{4}+512 x_{1}^{3}, \\
z_{2}\left(x_{2}\right) & =-244.140625 x_{2}^{6}+585.9375 x_{2}^{5}-468.75 x_{2}^{4}+125 x_{2}^{3}, \\
y^{*}\left(x_{1}, x_{2}\right) & = \begin{cases}z_{1}\left(x_{1}\right) z_{2}\left(x_{2}\right) & \text { in }(0,0.5) \times(0,0.8), \\
0 & \text { else },\end{cases} \\
u^{*}\left(x_{1}, x_{2}\right) & =y^{*}\left(x_{1}, x_{2}\right), \\
\xi^{*}\left(x_{1}, x_{2}\right) & =2 \max \left\{0,-\left|x_{1}-0.8\right|-\left|\left(x_{2}-0.2\right) x_{1}-0.3\right|+0.35\right\} .
\end{aligned}
$$

The data $f, y_{d}$ is set to

$$
f=-\Delta y^{*}-u^{*}-\xi^{*}, \quad y_{d}=y^{*}+\xi^{*}-\nu \Delta u^{*} .
$$

The parameter for the cost of the control is chosen as $\nu=1$. The solution $\left(y^{\star}, u^{\star}, \xi^{\star}\right)=\left(y^{*}, u^{*}, \xi^{*}\right)$ admits the regularity as stated in Remark 2.2. In fact, we even have $y^{\star}, u^{\star} \in C^{\infty}(\Omega) \cap H_{0}^{1}(\Omega)$ and $\xi^{\star}=-\lambda^{\star} \in W^{1, s}(\Omega)$ for $s \geq 1$.

On each refinement level in Algorithm 1, the optimization routine in line 2 (Algorithm 3) is employed with $\epsilon=10^{-6}, \gamma=10^{-3}$ and $\delta \gamma=1.5$. The solver for the penalized problem, Algorithm 2, is called with the parameter choices $c=1, \epsilon=10^{-6}, \zeta=0.5$ and $\kappa=10^{-4}$.

The solution calculated by our adaptive algorithm is shown in Figure 3. As $u^{\star}=y^{\star}$, we plot only $y_{h}^{\star}$. For visualization purposes, the values of the multiplier vector $\boldsymbol{\xi}_{\mathbf{h}}^{\star}=-\boldsymbol{\lambda}_{\mathbf{h}}^{\star}$ in the nodes are plotted on a coarser mesh. The size of the circles indicates the respective (nonnegative) value of $\boldsymbol{\xi}_{\mathbf{h}}^{\star}$. 

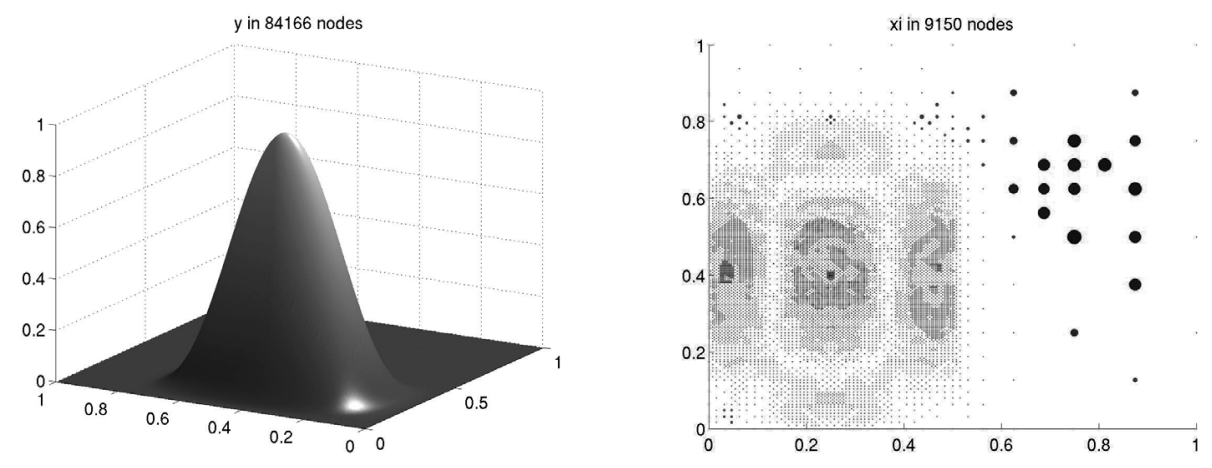

FiguRE 3. Solution graphs of the adaptive algorithm in Example 5.1, showing the state $y_{h}^{\star}$ $\left(=u_{h}^{\star}\right.$, left $)$ and the slack variable $\xi_{h}^{\star}\left(=-\lambda_{h}^{\star}\right.$, right $)$. For the sake of clarity, $\xi_{h}^{\star}$ is plotted on a coarser mesh.
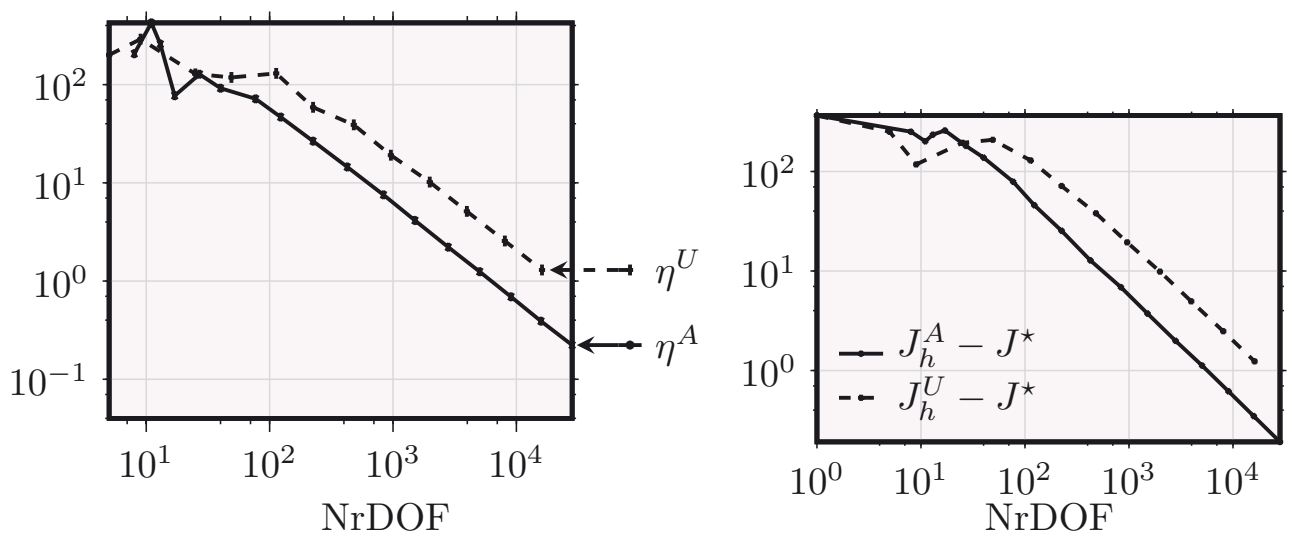

FiguRE 4. Comparison of convergence of the estimators for adaptive (solid lines) and uniform refinement (dashed lines) for Example 5.1.

Figure 4 shows on the left-hand side the comparison of convergence of the error estimators as a function of the number of degrees of freedom in logarithmic scale for adaptive versus uniform refinement. The corresponding error estimators are denoted by superscript " $A$ " for "adaptive" (solid lines) and " $U$ " for "uniform" refinement (dashed lines). The right-hand side of Figure 4 shows the convergence of $J_{h}^{A}-J^{\star}:=J_{h}\left(y_{h}^{\star}, u_{h}^{\star}\right)-J\left(y^{\star}, u^{\star}\right)$ for discrete solutions $y_{h}^{\star}, u_{h}^{\star}$ on the adapted mesh compared to the convergence of $J_{h}^{U}-J^{\star}:=J_{h}\left(y_{h}^{\star}, u_{h}^{\star}\right)-J\left(y^{\star}, u^{\star}\right)$ for solutions $y_{h}^{\star}, u_{h}^{\star}$ on uniform meshes.

All parts of the estimators converge to zero, and in the adaptive variant of the algorithm, the estimated error is smaller compared to the estimated error on a uniform mesh with the same number of degrees of freedom (NrDOF). Although the convergence speed (i.e., the slope of the graphs in Fig. 4) is not improved by adaptive mesh refinement owing to the high regularity of the solution, one can still observe a reduction of complexity when one aims at a certain accuracy in the objective value.

Table 1 is the convergence history of the adaptive refinement process including effectivity indices

$$
\frac{\eta_{h}^{A}}{\left|J_{h}^{A}-J^{\star}\right|}
$$


TABle 1. Convergence history of the adaptive method, AFEM levels 2, 6, 10, 14, 18 and 22 for Example 5.1 including effectivity indices.

\begin{tabular}{lccc}
\hline NrDOF & $\eta_{h}^{A}$ & $J_{h}^{A}-J^{\star}$ & Eff.Ind. \\
\hline 8 & $1.03 \mathrm{e}+02$ & $2.51 \mathrm{e}+02$ & 0.411 \\
27 & $6.38 \mathrm{e}+01$ & $1.81 \mathrm{e}+02$ & 0.352 \\
225 & $1.33 \mathrm{e}+01$ & $2.54 \mathrm{e}+01$ & 0.524 \\
2806 & $1.11 \mathrm{e}+00$ & $1.99 \mathrm{e}+00$ & 0.557 \\
28311 & $1.11 \mathrm{e}-01$ & $1.92 \mathrm{e}-01$ & 0.581 \\
235726 & $1.33 \mathrm{e}-02$ & $1.23 \mathrm{e}-02$ & 1.08 \\
\hline
\end{tabular}

TABle 2. Convergence history of the adaptive method, AFEM levels 2, 10, 17, 23 and 29 for Example 5.2 including effectivity indices.

\begin{tabular}{lccc}
\hline NrDOF & $\eta_{h}$ & $J_{h}^{\star}-J^{\star}$ & Eff.Ind. \\
\hline 18 & $5.51 \mathrm{e}-02$ & $1.19 \mathrm{e}-01$ & 0.463 \\
106 & $2.33 \mathrm{e}-03$ & $7.20 \mathrm{e}-03$ & 0.324 \\
1196 & $2.50 \mathrm{e}-04$ & $4.08 \mathrm{e}-04$ & 0.612 \\
13103 & $2.57 \mathrm{e}-05$ & $3.58 \mathrm{e}-05$ & 0.717 \\
133458 & $2.65 \mathrm{e}-06$ & $2.52 \mathrm{e}-06$ & 1.05 \\
\hline
\end{tabular}

on the respective AFEM levels $h$. Note that the value of $J^{\star}:=J\left(y^{\star}, u^{\star}\right)$ can be computed exactly from the solutions $y^{\star}, u^{\star}$ in this problem. The effectivity indices are smaller than 1 on coarse meshes, i.e., we underestimate the real difference of the objective values. When refining the mesh, the effectivity index increases to a value greater than 1 , which indicates that the error estimator becomes reliable. The same effect occurs in test computations of [8] when using the DWR approach for discretizing PDE problems or optimization problems with a PDE constraint (but without inequality constraints).

Example 5.2. We consider again $\mathcal{A}=-\Delta$ on the L-shaped domain $\Omega=(-1,0) \times(-1,1) \cup(0,1) \times(0,1)$ and define

$$
\begin{aligned}
y_{d}(x) & = \begin{cases}-1 & \text { if }|x| \geq \frac{1}{10} \\
1-100 x_{1}^{2}-50 x_{2}^{2} \text { else }\end{cases} \\
f(x) & =\frac{1}{2}+\frac{1}{2}\left(x_{1}-x_{2}\right), \\
\nu & =0.01 .
\end{aligned}
$$

On each refinement level in Algorithm 1, the optimization routine in line 2 (Algorithm 3) is employed with $\epsilon=10^{-6}, \gamma=10^{-2}$ and $\delta \gamma=1.5$. The solver for the penalized problem, Algorithm 2, is called with the parameter choices $c=0.1, \epsilon=10^{-6}, \zeta=0.5$ and $\kappa=10^{-4}$.

The solutions calculated by our adaptive algorithm are depicted in Figure 5.

In Figure 6, we show again the convergence of the error estimator in adaptive versus uniform refinement (left), as well as the convergence comparison of $J_{h}\left(y_{h}^{\star}, u_{h}^{\star}\right)-J\left(y^{\star}, u^{\star}\right)$ (right). The lack of regularity of the solution due to the non-convexity of $\Omega$ seems to favor adaptive refinement when compared to uniform refinement. In fact, we observe a significantly better convergence rate for AFEM in this case.

Table 2 lists the data from the plots in Figure 6 together with effectivity indices for different AFEM levels. The effectivity indices show once again that the error estimators are not reliable, but converge to 1 , similar to those in [8] for discretizing PDEs. The value of $J\left(y^{\star}, u^{\star}\right)$ used for the plot and the table is estimated by solutions on the adaptively generated mesh after additional uniform refinements. Finally, the adaptively 

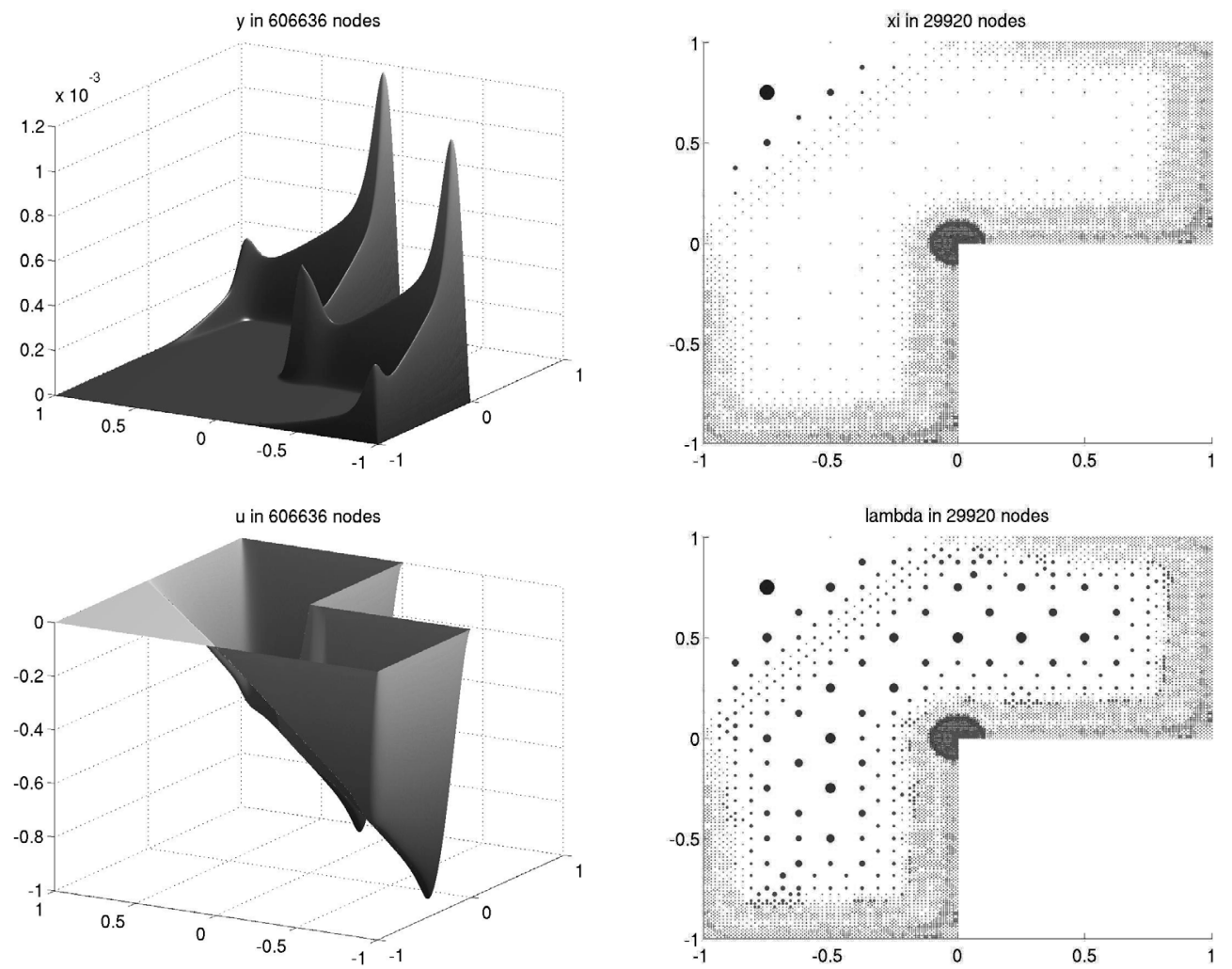

FIGURE 5. Solution graphs of the adaptive algorithm in Example 5.2, first row left state $y_{h}^{\star}$, right slack variable $\xi_{h}^{\star}$, second row left control $u_{h}^{\star}$ and right multiplier $\lambda_{h}^{\star}$.
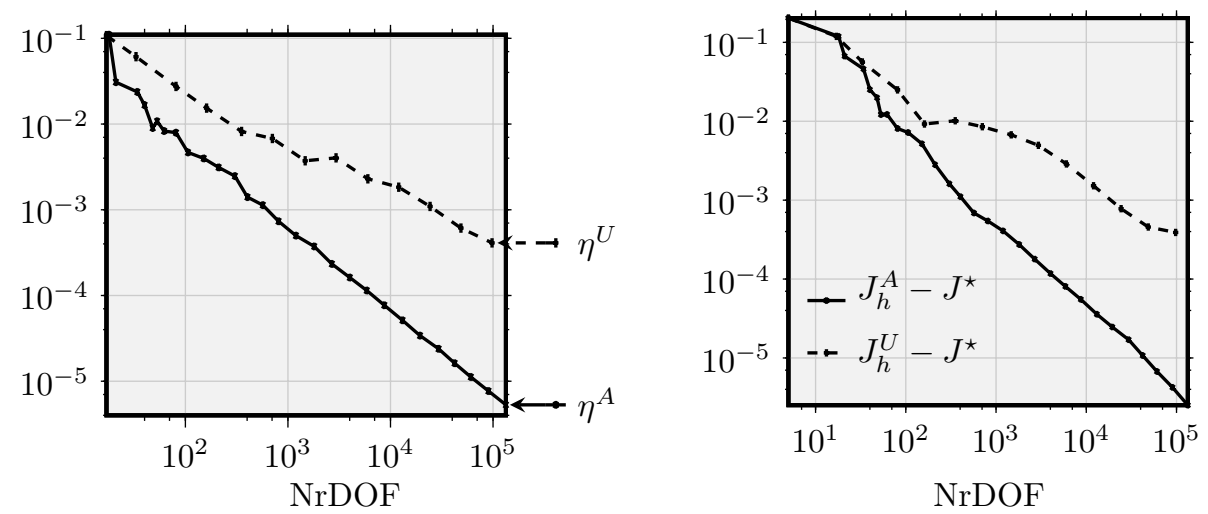

FIGURE 6. Comparison of convergence of the estimators for adaptive (solid lines) and uniform refinement (dashed lines) for Example 5.2.

generated mesh is depicted in Figure 7. We observe refinement in the non-convex corner as well as in the regions where $y_{h}^{\star}$ is steep. Further refinement is found near the boundary between active and inactive sets, which is due to the complementarity mismatch error. 


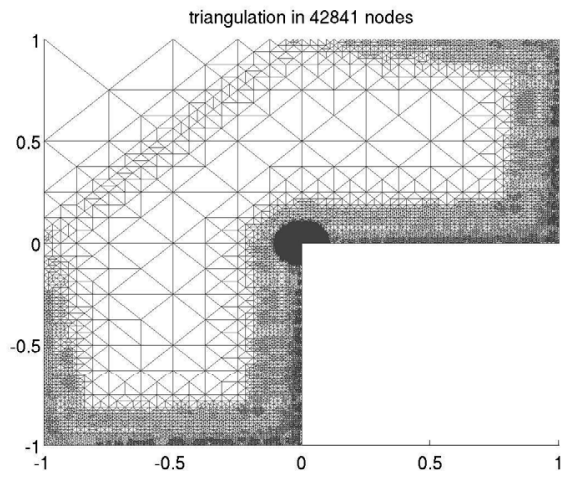

Figure 7. Adaptively refined mesh for Example 5.2.

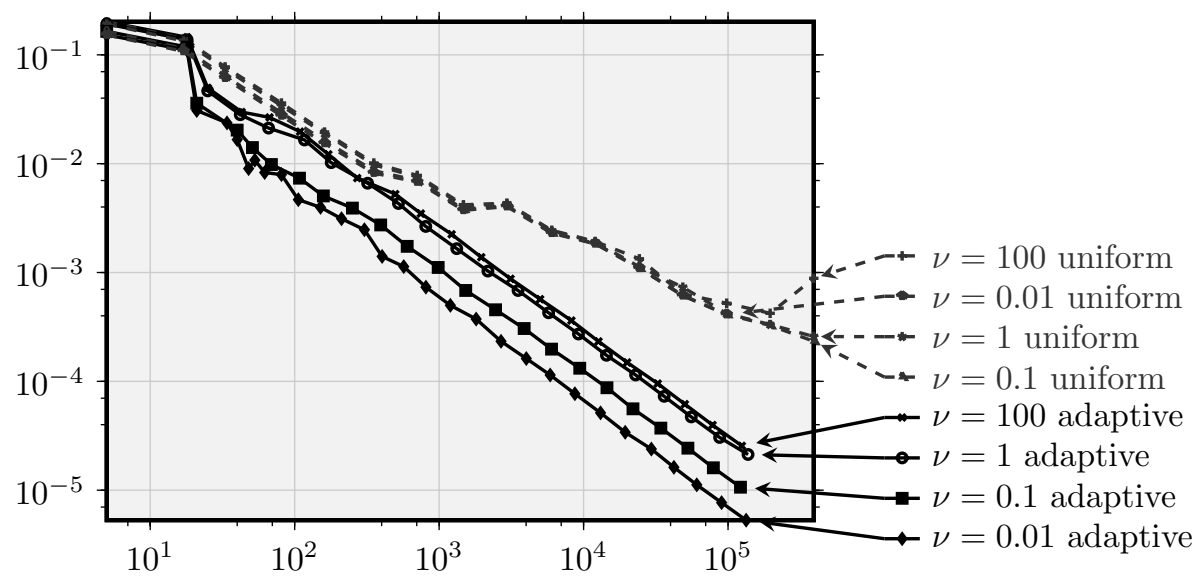

FiguRE 8. Comparison of convergence of the estimators for adaptive (solid lines) and uniform refinement (dashed lines) for Example 5.2 with different values of the control cost parameter $\nu$.

We also examined the behavior of our estimator for different values of the control cost $\nu$. In fact, the error estimator appears robust in this respect; see Figure 8.

\section{REFERENCES}

[1] K. Atkinson and W. Han, Theoretical Numerical Analysis: A Functional Analysis Framework. Springer (2009).

[2] M. Anitescu, P. Tseng and S.J. Wright, Elastic-mode algorithms for mathematical programs with equilibrium constraints: global convergence and stationarity properties. Math. Program 110 (2005) 337-371.

[3] E. Bänsch, Local mesh refinement in 2 and 3 dimensions. IMPACT Comput. Sci. Engrg. 3 (1991) $181-198$.

[4] V. Barbu, Optimal Control of Variational Inequalities. Pitman, Boston, London, Melbourne (1984).

[5] D. Braess, C. Carstensen and R.H.W. Hoppe, Convergence analysis of a conforming adaptive finite element method for an obstacle problem. J. Numer. Math. 107 (2007) 455-471.

[6] D. Braess, C. Carstensen and R.H.W. Hoppe, Error reduction in adaptive finite element approximations of elliptic obstacle problems. J. Comput. Math. 27 (2009) 148-169. 
[7] R. Becker, H. Kapp and R. Rannacher, Adaptive finite element methods for optimal control of partial differential equations: Basic concept. SIAM J. Control Optim. (2000).

[8] W. Bangerth and R. Rannacher, Adaptive Finite Element Methods for Differential Equations. Birkhäuser Verlag, Basel (2003).

[9] D. Braess, A posteriori error estimators for obstacle problems - another look. Numer. Math. 101 (2005) $415-421$.

[10] O. Benedix and B. Vexler, A posteriori error estimation and adaptivity for elliptic optimal control problems with state constraints. Comput. Optim. Appl. 44 (2009) 3-25.

[11] I. Babuška, J. Whiteman and T. Strouboulis, Finite Elements: An Introduction to the Method and Error Estimation. Oxford University Press (2011).

[12] C. Carstensen, An adaptive mesh-refining algorithm allowing for an $H^{1}$ stable $L^{2}$ projection onto Courant finite element spaces. Constructive Approximation 20 (2004) 549-564.

[13] M.C. Ferris and T.S. Munson, Interfaces to path 3.0: Design, implementation and usage. Comput. Optim. Appl. 12 207-227 (1999).

[14] F. Facchinei and J.S. Pang. Finite-Dimensional Variational Inequalities and Complementarity Problems, in vol. 1 of Springer Ser. Oper. Research. Springer (2003).

[15] A. Günther and M. Hinze, A posteriori error control of a state constrained elliptic control problem. J. Numer. Math. 16 (2008) $307-322$.

[16] M. Hintermüller and R.H.W. Hoppe, Goal-oriented adaptivity in control constrained optimal control of partial differential equations. SIAM J. Control Optim. 47 (2008) 1721-1743.

[17] M. Hintermüller and R.H.W. Hoppe, Goal-oriented adaptivity in pointwise state constrained optimal control of partial differential equations. SIAM J. Control Optim. 48 (2010) 5468-5487.

[18] M. Hintermüller and R.H.W. Hoppe, Goal-oriented mesh adaptivity for mixed control-state constrained elliptic optimal control problems, in vol. 15 of Appl. Numer. Partial Differ. Eq., edited by W. Fitzgibbon, Y.A. Kuznetsov, P. Neittaanmäki and J. Périaux. Comput. Methods Appl. Sci. Springer, Berlin-Heidelberg-New York (2010) 97-111.

[19] M. Hintermüller, M. Hinze and M.H. Tber, An adaptive finite-element Moreau-Yosida-based solver for a non-smooth CahnHilliard problem. Optim. Methods Software 26 (2011) 777-811.

[20] M. Hintermüller, K. Ito and K. Kunisch. The primal-dual active set strategy as a semismooth Newton method. SIAM J. Optim. 13 (2002) 865-888.

[21] M. Hintermüller and I. Kopacka. Mathematical programs with complementarity constraints in function space: C- and strong stationarity and a path-following algorithm. SIAM J. Optim. 20 (2009) 868-902.

[22] M. Hintermüller and I. Kopacka, A smooth penalty approach and a nonlinear multigrid algorithm for elliptic MPECs. Comput. Optim. Appl. 50 (2011) 111-145. DOI: 10.1007/s10589-009-9307-9.

[23] M. Hintermüller and T. Surowiec, A bundle-free implicit programming approach for a class of MPECs in function space. Preprint (2012).

[24] C. Johnson, Adaptive finite element methods for the obstacle problem. Math. Models Methods Appl. Sci. 2 (1992) $483-487$.

[25] D. Klatte and B. Kummer, Nonsmooth Equations in Optimization: Regularity, Calculus, Methods and Applications. Nonconvex Optim. Appl. Kluwer Academic (2002).

[26] D. Kinderlehrer and G. Stampacchia, An Introduction to Variational Inequalities and Their Applications. Academic Press, New York (1980).

[27] K. Kunisch and D. Wachsmuth. Path-following for optimal control of stationary variational inequalities. Comput. Optim. Appl. 51 (2012) 1345-1373.

[28] Z.Q. Luo, J.S. Pang and D. Ralph, Mathematical Programs with Equilibrium Constraints. Cambridge University Press (1996).

[29] W. Liu and N. Yan, A posteriori error estimates for distributed convex optimal control problems. Advances Comput. Math. 15 (2001) 285-309.

[30] B.S. Mordukhovich, Variational Analysis and Generalized Differentiation I: Basic Theory, vol. 330 of Grundlehren der mathematischen Wissenschaften. Springer (2006).

[31] B.S. Mordukhovich, Variational Analysis and Generalized Differentiation II: Applications, vol. 331 of Grundlehren der mathematischen Wissenschaften. Springer (2006).

[32] F. Mignot and J.P. Puel. Optimal control in some variational inequalities. SIAM J. Control Optim. 22 (1984) $466-476$.

[33] P. Neittaanmäki, J. Sprekels and D. Tiba, Optimization of Elliptic Systems: Theory and Applications. Springer Monogr. Math. Springer (2006).

[34] R.H. Nochetto, K.G. Siebert and A. Veeser, Pointwise a posteriori error control for elliptic obstacle problems. Numer. Math. 95 (2003) 163-195. DOI: 10.1007/s00211-002-0411-3.

[35] J.V. Outrata, M. Kočvara and J. Zowe, Nonsmooth Approach to Optimization Problems with Equilibrium Constraints: Theory, Applications, and Numerical Results, vol. 152 of Nonconvex Optim. Appl. Kluwer Academic Publishers (1998).

[36] D. Ralph. Global convergence of damped Newton's method for nonsmooth equations via the path search. Math. Oper. Res. 19 (1994) 352-389.

[37] S.I. Repin, A Posteriori Estimates for Partial Differential Equations. Radon Ser. Comput. Appl. Math. De Gruyter (2008).

[38] J.-F. Rodrigues, Obstacle Problems in Mathematical Physics. North-Holland, Amsterdam (1987).

[39] A. Rösch and D. Wachsmuth, A posteriori error estimates for optimal control problems with state and control constraints. Numer. Math. 120 (2012) 733-762. 
[40] H. Scheel and S. Scholtes, Mathematical programs with complementarity constraints: stationarity, optimality, and sensitivity. Math. Oper. Research 25 (2000) 1-22.

[41] A. Veeser, Efficient and reliable a posteriori error estimators for elliptic obstacle problems. SIAM J. Numer. Anal. 39 (2001) 146-167.

[42] R. Verfürth, A review of a posteriori error estimation and adaptive mesh-refinement techniques. Wiley-Teubner (1996).

[43] B. Vexler and W. Wollner, Adaptive finite elements for elliptic optimization problems with control constraints. SIAM J. Control Optim. 47 (2008) 509-534. 